\title{
Estimation of Myocardial Strain and Contraction Phase from Cine MRI using Variational Data Assimilation
}

\author{
Viateur Tuyisenge, Laurent Sarry, Thomas Corpetti, Elisabeth Innorta-Coupez, Lemlih Ouchchane, and Lucie Cassagnes
}

\begin{abstract}
This paper presents a new method to estimate left ventricle deformations using variational data assimilation that combines image observations from cine MRI and a dynamic evolution model of the heart. The main contribution of the model is that it embeds parameters modeling the contraction / relaxation process. It estimates myocardial motion and contraction parameters simultaneously, providing accurate complementary information for diagnosis. The method was applied to synthetic datasets with known ground truth motion and to 47 patients MRI datasets acquired at three slice locations (base, mid-ventricle and apex). Radial and circumferential strain components were compared to those obtained with a reference tag tracking software, exhibiting good agreement with intraclass correlation coefficients (ICC) above 0.8. Results were also evaluated against wall motion score indices used to assess cardiac kinetics in clinical practice. The assimilation process overcame issues caused by temporal artifacts as a result of the dynamic model, compared to using the observation term alone. Moreover we found that the new dynamic model, consisting of a piecewise transport model acting independently on systole and diastole performed better than the standard continuous transport model, which oversmooths temporal variations. Estimated strain and contraction parameters significantly correlated to clinical scores, making them promising features for diagnosing not only hypokinesia but also dyskinesia.
\end{abstract}

Index Terms-Myocardial motion, myocardial strain, contraction phase, MRI, data assimilation, cardiac dyskinesia.

\section{INTRODUCTION}

$\mathrm{T}$ HERAPEUTICS in heart failure are not unique as far as left ventricular systolic function is concerned [1], [2]. Function may be assessed globally by measuring Left Ventricular Ejection Fraction (LVEF). 2/3D echocardiography is used routinely to provide LVEF and to follow-up patients while Magnetic Resonance Imaging (MRI) has become the gold standard for reliability and reproducibility of ventricular volumes, thickening, motion and mass as well as for anatomic and functional analysis [3]. Echocardiography and MRI are also used for precise analysis of left ventricular kinetics.

The addition of myocardial tagging to cine MRI enables more sensitive detection of early myocardial wall dysfunction and makes it possible to estimate of myocardial strain, i.e. changes in shape during systolic contraction [4]. The method proposed here is an alternative to tag tracking techniques [5], [6], [7], that also works on non-tagged cine MRI acquisitions. Accurate estimation of strain is essential to clinical practice as attested by a recent prospective study that found strain analysis to be sensitive and reliable for detecting myocardial dysfunction, and to provide objective and quantitative indexes superior to the visual analysis of left ventricular motion abnormalities [8].

Non-invasive image-based analysis and quantification of cardiac motion yields key information on how pathology affects local and

Viateur Tuyisenge, Laurent Sarry and Lemlih Ouchchane are with Clermont Université, Université d'Auvergne, ISIT UMR 6284 UdA-CNRS, ClermontFerrand, France.

Elisabeth Innorta-Coupez and Lucie Cassagnes are with Pôle de Radiologie et d'Imagerie Médicale, CHU Gabriel Montpied, Clermont-Ferrand, France.

Thomas Corpetti is with COSTEL-LETG UMR 6554 CNRS, Université de Rennes 2, Rennes, France.

Copyright (c) 2010 IEEE. Personal use of this material is permitted. However, permission to use this material for any other purposes must be obtained from the IEEE by sending a request to pubs-permissions@ieee.org. global deformation of the myocardium and its responses to a given therapy. The estimation of myocardial deformations helps study and detect regions with abnormal contraction to guide treatment for recovery. It includes a comprehensive study of structural or architectural heart abnormalities, which provides an essential prognostic approach for therapeutic decisions, such as the implantation of defibrillators for cardiac resynchronization therapy or the adaptation of doses or treatments such as beta-blockers converting enzyme inhibitors.

The past few years have seen an intensive drive to develop methods to track the myocardium in imaging modalities ranging from ultrasound (US) [9], [10], [11] to magnetic resonance imaging (cine MRI or tagged MRI) [6], [12], [13] and SPECT [14]. However, accurate cardiac motion estimation is still an unresolved challenge due to low spatio-temporal imaging resolutions and the complexity of cardiac biomechanics [15], [16], [17]. An array of approaches aiming to extract cardiac contraction parameters and deformation fields from images and the underlying dynamics of the heart are reviewed in [18]. To improve accuracy, knowledge about cardiac contraction can be used to constrain the solution in a data assimilation framework.

Variational data assimilation was initially derived from optimal control theory to recover a state variable trajectory, driven by an approximatively known dynamical law, from a sequence of measurements [19]. This technique has been used with complex partial differential equations (PDEs) to track curves and estimate physical parameters (motion, temperature, etc) in geosciences and computer vision disciplines [20], [21], [22], [23], [24]. Technical details can be found in [25], [26], [27], [28]. To improve cardiac disease diagnosis and therapy planning, researchers have extended the technique to cardiac medical images to benefit from the robustness and accuracy of this estimation process. Sainte-Marie et al. [29] and Sermesant et al. [30] used data assimilation to estimate local cardiac contractility from synthetic cardiac data. Sundar et al. [31], Delingette et al. [32] and Marchesseau et al. [33] estimated myocardial motion and contractility parameters of left and right ventricles using data assimilation and a full electromechanical model of the heart and reported promising results in the field of cardiac personalization.

The goal here was not to design a new physiological model of the heart, but to propose a simple evolution model designed to drive the measurement of myocardial motion without imposing strong constraints over the displacement field. The brief was to develop a generic model with enough degrees of freedom to not hide defects in cardiac kinetics due to various pathologies. To this end, a transport equation parameterized by the contraction phase was used as a dynamic evolution model in a variational data assimilation framework.

Our model brings three novel contributions over previous works. (1) It includes contraction parameters and makes displacement field estimation more accurate. Indeed, invariance assumption, usually embedded in dynamics models, tends to oversmooth temporal variations in the transported flow field values. It is geared to uniformly accelerated movements, but is unsuitable for cardiac dynamics. Therefore, an asymmetric square wave function is used to account for the sign of the contraction, essentially by piecewisely and independently smoothing the contraction and relaxation phases. (2) Flow fields and contraction 
parameters are simultaneously estimated as complementary features to diagnose cardiac dyskinesia. (3) The method includes observation terms that preserves motion discontinuities. The method was applied to synthetic and 47 real cine MRI datasets, its validity was studied against a reference method for MRI tag tracking and informational value was assessed towards clinical expertise.

\section{MEthods AND MATERIALS}

\section{A. Image Data Description}

1) MRI sequence simulation: A synthetic dataset was generated to evaluate the accuracy of the proposed methods. The images of the simulated MRI sequence were obtained from the image at the initial time and a displacement field using a transport model. A third order finite differencing scheme was used as it does not suffer from the artifacts observed for lower order schemes as boundary rebound effect or diffusion. Figure 1 illustrates the results of the simulation process from a synthetic displacement field made of a radial contraction component and a circumferential torsion component with two-fold lower in amplitude (Fig. 1b) applied inside a mask delimiting myocardium on the first image (Fig. 1a). Temporal motion variations are assumed to follow a sinusoidal pattern. To simulate spatial contraction discrepancies, parameters $\varphi$ and $a$ vary angularly as a sinusoidal wave around the myocardium (Fig. $4 \mathrm{~b}$ and $4 \mathrm{c}$ ). Four image datasets are generated with the following ranges for $\varphi$ and $a$ : dataset \#1 $\varphi \in[-\pi / 6, \pi / 6]$ and $a \in[0.4,0.6]$, dataset \#2 $\varphi \in[-\pi / 6, \pi / 6]$ and $a \in[0.15,0.35]$, dataset $\# 3 \varphi \in[-\pi / 4, \pi / 12]$ and $a \in[0.4,0.6]$ and dataset $\# 4 \varphi \in[-\pi / 6, \pi / 6]$ and $a \in[-0.1,0.1]$. Figures $1 \mathrm{c}$ and $1 \mathrm{~d}$ illustrate the resulting deformation between end systole and end diastole when applied to the initial image.

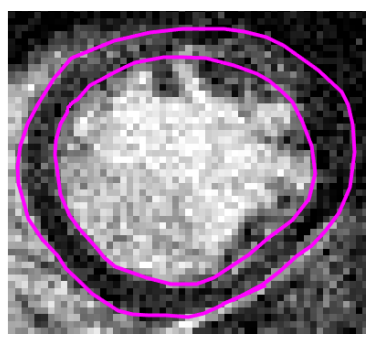

(a)

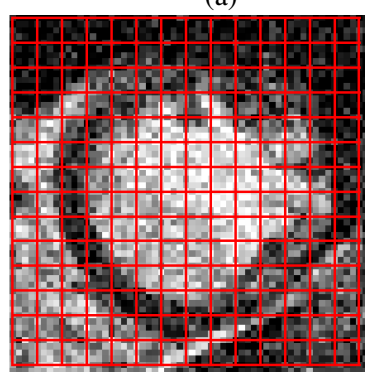

(c)

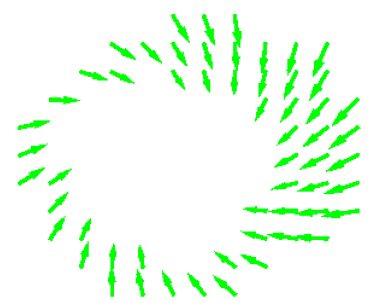

(b)

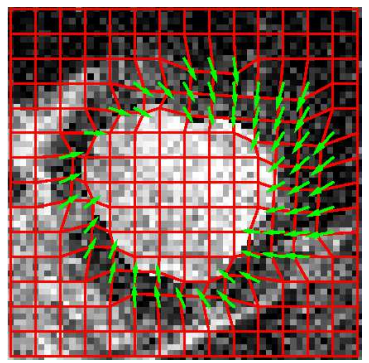

(d)
Fig. 1: Simulated image dataset \#1. (a) Myocardium delineated on the reference cine MRI frame at end diastole and (b) the synthetic displacement field. A regular grid overlaid on the reference cine MRI frame (c) underwent myocardial deformation when overlaid on the frame at end systole (d).

To test the efficiency and the robustness of the assimilation dynamical model, we created artifacts similar to time varying occultation. This is the case for dark band artifacts [34] (DBA), even if they are known to occur at $3 \mathrm{~T}$ rather than $1.5 \mathrm{~T}$. These are caused by blood or myocardial motion, which causes loss in image intensity due to a breakdown in the steady state signal. Indeed flow may bring the image to off-resonance regions with $B_{0}$ inhomogeneities. Four image sequences are generated with artefacts simulated as horizontal or vertical dark bands, placed in the middle or at one quarter of the image size, appearing every four images of the sequence by modulating the intensity with a spatial sinusoid of maximal attenuation of $50 \%$ (Fig. 5a).

The influence of noise has also been tested by adding zero mean Gaussian noise with different standard deviations to the images of the simulated sequence. Multiple repeats have been generated in order to demonstrate the stability of the estimation.

2) Real MRI sequence acquisition and preprocessing: Cine and tagged MRI image sequences were acquired for 47 patients with abnormal kinetics (hypokinesia, akinesia or dyskinesia). MRI was part of a post-myocardial infarction protocol. MRI sequences were acquired with an Avanto 1.5T system (Siemens, Forchheim, Germany) using a cine trueFISP sequence in short axis view at basal, mid-ventricular and apical slice locations with and without tagging pattern (grid spacing of $7 \mathrm{~mm}$ ). Spatial and temporal resolutions were not the same for cine and tagged MRI, but were kept constant for every scans of every patient. The settings were: pixel dimensions $1.8 \mathrm{~mm} \times 1.8 \mathrm{~mm}$, slice thickness of $7 \mathrm{~mm}$ and temporal step of $31 \pm 6 \mathrm{~ms}$ with prospective gating for cine MRI; pixel dimensions $2.0 \mathrm{~mm} \times 1.3 \mathrm{~mm}$, slice thickness of $6 \mathrm{~mm}$ and temporal step of $34 \pm 4 m s$ with prospective gating for tagged MRI. Image numbers ranged from 19 to 36 for one cardiac cycle of length of time $T$. As our goal was to stay as close as possible to the the clinical protocol of left-ventricular function evaluation from cine or tagged MRI [35], no out-of-plane motion compensation method was used.

Sequences were first spatially registered to compensate for offsets arising from a lack of breath-hold reproducibility (two points handselected in the middle of the LV cavity and at the junction to the $\mathrm{RV}$ in order to center and reorient the images). The accuracy of the registration process at a few millimeters is good enough to compare values averaged over AHA standard sectors [36]. Image sequences are then temporally interpolated to get the same number of frames with cubic interpolation. Temporal comparison between scans was valid because there was only two minutes between cine and tagged MR scans, and patients were not arythmic with betablocker treatment, heart rate can be assumed stable. Results of cardiac displacement and strain estimated from cine MRI using three variational methods were cross-compared against the results computed on tagged MRI images using inTag software ${ }^{1}$.

\section{B. Data Assimilation Overview}

We present a method for cardiac motion and contraction parameter estimation using variational data assimilation. Data assimilation was chosen because it incorporates a dynamical model that provides temporal coherence and makes results robust to poor quality and potentially incomplete and noisy data.

In this framework, the transport equation which has found use as a default dynamical model for temporal regularization of displacement fields in satellite meteorological applications is replaced by a parameterized transport equation accounting for the nature of the contraction movement [37], [24]. To the best of our knowledge, this is the first time that this kind of model is applied to cardiac imaging data.

The model is introduced below and the mathematical principles of data assimilation used for estimating model parameters are introduced.

${ }^{1}$ inTag is an open-source OsiriX plugin developed by the CREATIS laboratory (http://www.creatis.insa-lyon.fr/inTag/). 


\section{Dynamic Evolution Model}

The transport equation based on fluid flow laws used by different authors for motion estimation on meteorological data overly smoothes the estimated fields, especially when direction is changing rapidly, see [21], [22], [24] and references therein. To handle the case of cardiac dynamics, we propose a parameterized transport equation. An asymmetric square wave function accounting for the sign of myocardium contraction is embedded into the standard transport equation, which leads to the following dynamic model:

$$
\mathbb{M}(\mathcal{X})=\operatorname{sgn}(\sin (\omega t+\varphi(\mathbf{x}, t))+a(\mathbf{x}, t)) \mathbf{v}^{\mathrm{T}}(\mathbf{x}, t) \nabla \mathcal{X}(\mathbf{x}, t),
$$

where $\operatorname{sgn}$ is the sign function, $\mathcal{X}=(\mathbf{v}(\mathbf{x}, t), \varphi(\mathbf{x}), a(\mathbf{x}))^{\mathrm{T}}$ is the state vector, $\mathbf{v}(\mathbf{x}, t)=(u(\mathbf{x}, t), v(\mathbf{x}, t))$ is the displacement field, $\omega=$ $2 \pi / T$ is the cardiac pulsation, and $\varphi(\mathbf{x})$ and $a(\mathbf{x})$ are phase angle and temporal asymmetry respectively. As illustrated in Fig. $2, a \in[-1,1]$ implicitly defines the ratio between the durations of systole $S$ and diastole $D$

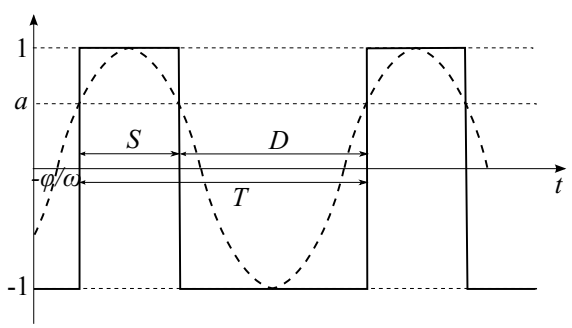

Fig. 2: Function modeling contraction / relaxation movement of the heart. It is based on a $s g n$ function (blue) applied to a sinus function (red) of phase $\varphi$ and offset $a . S, D$ and $T$ stand for systolic, diastolic and full cardiac periods.

In fact, $\varphi$ gives the phase of the contraction and $a$ acts on the symmetry of the cardiac cycle (for instance $a=0.5$ corresponds to $D / S=2$ ). The assimilation process adjusts their values to synchronize the asymmetric square wave function with the cardiac cycle (positive for contraction and negative for relaxation). Its principle is detailed in the next paragraph.

\section{Mathematical Formulation for Data Assimilation}

We are dealing with a problem that seeks to recover a system's state $\mathcal{X}(\mathbf{x}, t)$ abiding to a given dynamical law that governs system evolution. The dynamical system is defined by the non-linear operator $\mathbb{M}$ depending on state variables. The measurements $\mathcal{Y}$ (commonly known as observations) are assumed to be available at some discrete times and are measured through a non-linear operator $\mathbb{H}$ that belongs to a Hilbert space. This operator links the system state variables to the observation function. Given these different data, assimilation aims to produce accurate estimates of the current and future states of the dynamic system by solving the following system of three equations:

$$
\left\{\begin{array}{l}
\frac{\partial \mathcal{X}}{\partial t}(\mathbf{x}, t)+\mathbb{M}(\mathcal{X})(\mathbf{x}, t)=\nu_{m}(\mathbf{x}, t) \\
\mathcal{Y}(\mathbf{x}, t)=\mathbb{H}(\mathcal{X})(\mathbf{x}, t)+\nu_{o}(\mathbf{x}, t) \\
\mathcal{X}(\mathbf{x}, 0)=\mathcal{X}_{b}(\mathbf{x})+\nu_{b}(\mathbf{x}, 0)
\end{array}\right.
$$

where $(\mathbf{x}, t)$ are the spatio-temporal variables, $\boldsymbol{\mathcal { X }}_{b}$ is the background (a priori knowledge of the state vector) and $\nu_{m}, \nu_{o}$ and $\nu_{b}$ are the uncertainties of dynamic evolution, observation and background information respectively. They are associated to definite positive endomorphisms $\mathbf{Q}, \mathbf{R}$ and $\mathbf{B}$ referred to as error covariance tensors. The system (2) is solved by minimizing the following functional:

$$
\begin{aligned}
E(\mathcal{X})= & \int_{\mathbf{x}, t}\left(\frac{\partial \mathcal{X}}{\partial t}+\mathbb{M}(\mathcal{X})\right)^{\mathrm{T}} \mathbf{Q}^{-1}\left(\frac{\partial \mathcal{X}}{\partial t}+\mathbb{M}(\mathcal{X})\right) d \mathbf{x} d t \\
& +\int_{\mathbf{x}, t}(\mathcal{Y}(\mathbf{x}, t)-\mathbb{H}(\mathcal{X})(\mathbf{x}, t))^{T} \mathbf{R}^{-1}(\mathcal{Y}(\mathbf{x}, t)-\mathbb{H}(\mathcal{X})) d \mathbf{x} d t \\
& +\int_{\mathbf{x}}\left(\mathcal{X}_{b}-\mathcal{X}_{0}\right)^{\mathrm{T}} \mathbf{B}^{-1}\left(\mathcal{X}_{b}-\mathcal{X}_{0}\right) d \mathbf{x} .
\end{aligned}
$$

A common way to optimize this cost function is to compute the gradient of $E$ and use a gradient descent algorithm. Unfortunately a direct computation of this gradient is too expensive from a computational point of view since it would require to integrate the dynamical system along all possible perturbations of $\mathcal{X}$, which in unfeasible in practice for large system states. An alternative approach is the adjoint technique [20]. We introduce the adjoint variables $\lambda$ that express the errors of the dynamic model:

$$
\lambda=Q^{-1}\left(\frac{\partial \mathcal{X}}{\partial t}+\mathbb{M}(\mathcal{X})\right)
$$

where $\left(\frac{\partial \mathbb{M}}{\partial \tilde{\mathcal{X}}}\right)$ and $\left(\frac{\partial \mathbb{H}}{\partial \tilde{\mathcal{X}}}\right)$ are the linear tangent operators of $\mathbb{M}$ and $\mathbb{H}$ respectively. The linear tangent of an operator $\mathbb{A}$ is the directional derivative of the operator (the Gâteaux derivative):

$$
\left(\frac{\partial \mathbb{A}}{\partial \widetilde{\mathcal{X}}}\right)(d \mathcal{X})=\lim _{\beta \rightarrow 0} \frac{\mathbb{A}(\tilde{\mathcal{X}}+\beta d \mathcal{X})-\mathbb{A}(\widetilde{\mathcal{X}})}{\beta},
$$

and $\left(\partial_{\mathcal{X}} \mathbb{M}\right)^{*}$ and $\left(\partial_{\mathcal{X}} \mathbb{H}\right)^{*}$ their adjoint operators. The adjoint $\mathbb{A}^{*}$ of a linear operator $\mathbb{A}$ on a space $\mathcal{D}$ is such as:

$$
\forall x_{1}, x_{2} \in \mathcal{D},<\mathbb{A} x_{1}, x_{2}>=<x_{1}, \mathbb{A}^{*} x_{2}>.
$$

It can be shown that estimating the gradient of $E$ with respect to the adjoint variables $\lambda$ leads to a retrograde integration of an adjoint evolution model that takes into account the observations [20]. Once the adjoint variables $\lambda$ are estimated, one can recover the system state $\mathcal{X}$ using relation (4).

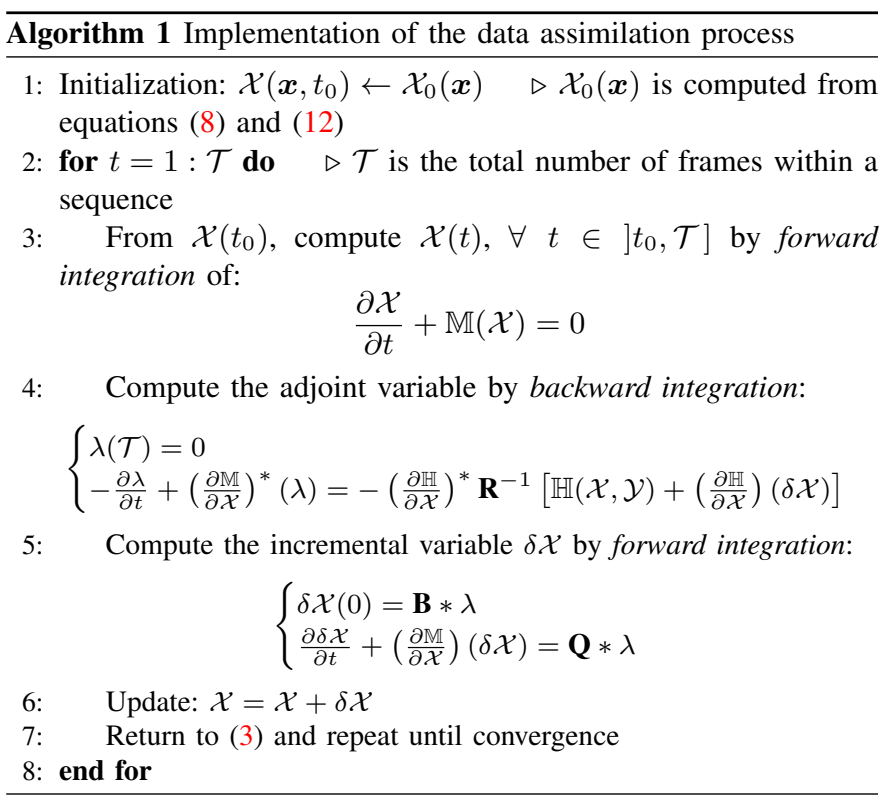

Intuitively, the adjoint variables $\lambda$ contain information about on discrepancy between observations and dynamic model. They are computed from a current solution with backward integration [21], [22] that encompasses both the observations and the dynamic operators. As described in algorithm 1, this deviation indicator between the 
observations and the model is then used to refine the initial condition (step 6) and to recover system state through an imperfect dynamic model where errors are modeled by $\mathbf{Q}$ (step 5). Note that if the dynamic is perfect, the associated error covariance $\mathbf{Q}$ is zero and the algorithm only refines the initial condition. For more informations, all computational details are given in [20].

In this paper we suggest to rely on this formalism to estimate myocardial strain and contraction phase introduced in the previous paragraph. In the following discussions, the standard transport model without the $\operatorname{sgn}$ function is referred to as DASS1 and our model (1) as DASS2. We now discuss the observation operator.

\section{E. Observation Models}

The observation operator on the displacement field relies on the optical flow constraint equation [38], [39], [40], [41], [42] combined with a non-linear regularizer [43], [44], [45], [46], that acts as both quadratic and total variation smoothing and preserves myocardial motion discontinuities [47], [48] (OBS). Displacement field $\mathbf{v}$ is the minimum of the following functional energy:

$$
\mathbf{v}(\mathbf{x}, t)=\operatorname{argmin} \int_{\Omega}\left[\left(\nabla I . \mathbf{v}+I_{t}\right)^{2}+\alpha \Phi(\|\nabla \mathbf{v}\|)\right] d \Omega,
$$

where $I$ denotes the intensity measured at position $(\mathbf{x}, t)$ on the image domain $\Omega, \nabla$ the gradient operator, $I_{t}$ the temporal derivatives, $\alpha$ is weight on the regularisation term and $\Phi$ is a function applied to the displacement gradient in order to preserve myocardial motion discontinuities.

After solving the Euler-Lagrange equation associated to the functional (7) and a mathematical simplification, we have $\Phi^{\prime \prime}(\|\nabla \mathbf{v}\|) \mathbf{v}_{\eta \eta}+\frac{\Phi^{\prime}(\|\nabla \mathbf{v}\|)}{\|\nabla \mathbf{v}\|} \mathbf{v}_{\xi \xi}=2 \alpha\left(\nabla I . \mathbf{v}+I_{t}\right) \nabla I$, where $\boldsymbol{\eta}$ and $\boldsymbol{\xi}$ define the Gauge coordinate system illustrated in Fig. 3a. For displacement coordinate $u, \boldsymbol{\eta}=\frac{1}{\|\nabla u\|}\left(u_{x} u_{y}\right)^{\mathrm{T}}$ is the normalized gradient of $u$ and $\boldsymbol{\xi}$, orthogonal to $\boldsymbol{\eta}$ i.e $\boldsymbol{\xi}=\frac{1}{\|\nabla \boldsymbol{u}\|}\left(-u_{y} u_{x}\right)^{\mathrm{T}}$, is tangential to the isointensity curves of $u$. The same definitions hold for coordinate $v$.

Different functions $\Phi$ (quadratic, total variation, etc.) have been proposed in the literature to weight the diffusion process in the normal direction $\eta$ and in the tangential direction $\xi$ (see for instance [49]). The key point is to do isotropic diffusion in regions with low amplitude motion gradients, and tangential diffusion elsewhere. As these two extreme cases cannot be perfectly handled by a unique function $\Phi$, we proposed in [48] to fix independent weights for $\boldsymbol{\eta}$ and $\boldsymbol{\xi}$ directions in the following way:

$$
g(\|\nabla \mathbf{v}\|) \mathbf{v}_{\eta \eta}+\mathbf{v}_{\xi \xi}=2 \alpha\left(\nabla I . \mathbf{v}+I_{t}\right) \nabla I,
$$

where $g($.$) denotes the Gaussian kernel g(\|\nabla \mathbf{v}\|)=$ $\frac{1}{2 \pi k} \exp \left(-\frac{\|\nabla \mathbf{v}\|^{2}}{2 k}\right) \cdot \quad \mathbf{v}_{\eta \eta}=\frac{\partial^{2} \mathbf{v}}{\partial \eta^{2}}=\nabla(\nabla \mathbf{v} \cdot \boldsymbol{\eta}) \cdot \boldsymbol{\eta}$ and $\mathbf{v}_{\xi \xi}=\frac{\partial^{2} \mathbf{v}}{\partial \xi^{2}}=\nabla(\nabla \mathbf{v} \cdot \boldsymbol{\xi}) \cdot \boldsymbol{\xi}$ are the second-order Gauge derivatives that can be obtained as the product of gradients in $\boldsymbol{\eta}$ and $\boldsymbol{\xi}$ directions and the Hessian matrix. For instance for coordinate $u$, we get:

$$
\begin{gathered}
u_{\eta \eta}=\frac{1}{u_{x}^{2}+u_{y}^{2}}\left(\begin{array}{ll}
u_{x} & u_{y}
\end{array}\right)\left(\begin{array}{ll}
u_{x x} & u_{x y} \\
u_{y x} & u_{y y}
\end{array}\right)\left(\begin{array}{l}
u_{x} \\
u_{y}
\end{array}\right), \\
u_{\xi \xi}=\frac{1}{u_{x}^{2}+u_{y}^{2}}\left(\begin{array}{ll}
u_{y} & -u_{x}
\end{array}\right)\left(\begin{array}{ll}
u_{x x} & u_{x y} \\
u_{y x} & u_{y y}
\end{array}\right)\left(\begin{array}{c}
u_{y} \\
-u_{x}
\end{array}\right) .
\end{gathered}
$$

After performing the matrix product above, we get the following equations:

$$
\begin{aligned}
& u_{\eta \eta}=\frac{u_{x}^{2} u_{x x}+2 u_{x} u_{x y} u_{y}+u_{y}^{2} u_{y y}}{u_{x}^{2}+u_{y}^{2}}, \\
& u_{\xi \xi}=\frac{u_{x}^{2} u_{y y}-2 u_{x} u_{x y} u_{y}+u_{y}^{2} u_{x x}}{u_{x}^{2}+u_{y}^{2}} .
\end{aligned}
$$

For the displacement field $\mathbf{v}$, the observation vector $\mathcal{Y}_{\mathbf{v}}$ is given by the spatio-temporal gradient $\nabla_{3} I(\mathbf{x}, t)=\left(\nabla I(\mathbf{x}, t) \quad I_{t}(\mathbf{x}, t)\right)^{\mathrm{T}}$. The observation operator $\mathbb{H}_{\mathbf{v}}$ linking the observations $\mathcal{Y}_{\mathbf{v}}$ to the unknown displacement field $\mathbf{v}$, is obtained by putting together the left-hand side and right-hand side of equation (8):

$$
\begin{aligned}
\mathbb{H}_{\mathbf{v}}\left(\mathbf{v}, \mathcal{Y}_{\mathbf{v}}\right)(\mathbf{x}, t)= & (\nabla I(\mathbf{x}, t) \cdot \mathbf{v}(\mathbf{x}, t)) \nabla I(\mathbf{x}, t)+I_{t} \nabla I(\mathbf{x}, t) \\
& -\frac{1}{2 \alpha}\left(\begin{array}{cc}
\triangle_{g, \eta, \xi} u & 0 \\
0 & \triangle_{g, \eta, \xi} v
\end{array}\right) \mathbf{v}(\mathbf{x}, t)
\end{aligned}
$$

where $\triangle_{g, \eta, \xi}=g(\|\nabla \cdot\|) \frac{\partial^{2} \cdot}{\partial \eta^{2}}+\frac{\partial^{2}}{\partial \xi^{2}}$.

To constrain values of contraction parameters $a$ and $\varphi$, the observation $\mathcal{Y}_{a, \varphi}$ measures the synchronization between the observed motion and the sinusoidal signal of the dynamical model. The radial component of displacement is the one that shows the most perfect oscillation, comparable with a sinusoid function. Thus the observation operator is built as the opposite of the negative correlation between the normalized radial component $\Lambda$ of the background displacement and the sinusoid of phase $\varphi$ and offset $a$ :

$$
\mathbb{H}_{a, \varphi}\left(a, \varphi, \mathcal{Y}_{a, \varphi}\right)(\mathbf{x}, t)=-\frac{\Lambda-\bar{\Lambda}}{\sigma_{\Lambda}}[\sin (\omega t+\varphi(\mathbf{x}, t))+a(\mathbf{x}, t)],
$$

where $\bar{\Lambda}$ and $\sigma_{\Lambda}$ are the mean and standard deviation of $\Lambda$ respectively. The resulting linear tangent operators and adjoint models are given in appendix A.

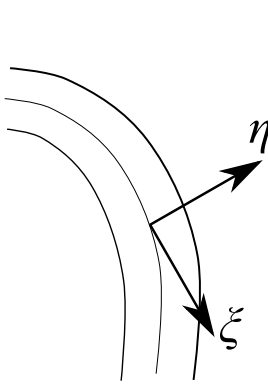

(a)

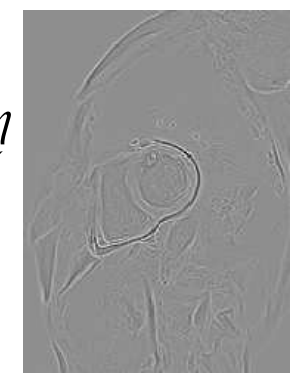

(b)

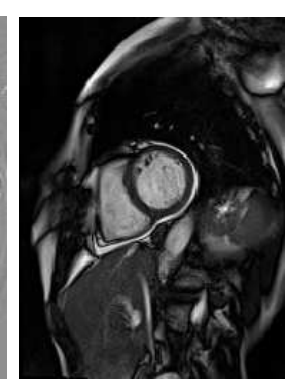

(c)
Fig. 3: (a) Illustration of a local first-order Gauge coordinate system; (b) the resulting second-order Gauge derivative $I_{\eta \eta}$ applied on a cineMRI image (c).

The covariance matrices $\mathbf{R}, \mathbf{Q}$, and $\mathbf{B}$ that represent errors in observation, evolution model and background, respectively, have to be defined to avoid the influence of observation noise on the computed solution. To this end, we define the observation covariance matrix as $\mathbf{R}(\mathbf{x})=\left(1-\exp ^{-\frac{\left\|\nabla_{3} I(\mathbf{x}, t)\right\|^{2}}{\sigma^{2}}}\right)$, where $\sigma$ is the standard deviation of the spatio-temporal gradient [50]. In practice, covariance matrix $\mathbf{Q}$ acts on the temporal regularization of the variations in state variables; if too small, then noisy variations could occur, or oversmoothing otherwise. It can be learnt in a training process on real data by minimizing the error between the estimated displacements and a ground truth. To this end, the reference displacement field given by inTag for one patient dataset was compared against the displacement field estimated with our method: minimum errors were obtained for variances of 10 and 0.1 for $(u, v)$ and $(a, \varphi)$ respectively. 
This patient was excluded from the database used for evaluation. Matrix $\mathbf{B}$ is chosen to be identity. All numerical details required for implementation are given in appendix B.

Note that motion outside the myocardial tissue does not completely fit the dynamical model, especially for the blood pool where turbulences may happen, but the observation operator applies everywhere. As a result displacements may be less temporally coherent outside myocardium, but they do not interfere with the measurements inside. To limit the influence of the exterior of the myocardium, a rectangular ROI is manually delimited at the beginning of the process. An automatic Otsu thresholding method provides a rough mask for myocardium pixels that look darker than blood pool. Since this simple processing is not accurate enough to provide a perfect delineation of endocardial and epicardial borders, we use a $90^{t h}$ percentile for the radial component (positive) and $10^{\text {th }}$ percentile for the circumferential component (negative) of strain instead of averaging strain values in order to exclude points outside myocardial region supposed to be negligible in amplitude.

\section{F. Statistical protocol for evaluation}

1) Analysis of simulated data: We assessed the relative robustness of the methods OBS, DASS1 and DASS2 with respect to deformation, level of noise and DBA as described in the simulation part. We used mixed linear model analyses, with a particular focus on the first order interactions between each of these three main effects and methods. We further performed multiple comparison procedures following Tukey method to detail these possible interactions and ease result interpretation.

2) Analysis of real data: The validity of the methods OBS, DASS1 and DASS2 was studied assessing the concordance with inTag, considered as the gold standard method. First, we sought for systematic difference between strain or displacement values between the methods by performing a discordance test, using a paired non parametric signed rank test. Then, the concordance between each method and inTag was estimated using ICC. These analyses were completed with Bland and Altman plots.

The clinical informational value of strain and displacement as measured by all methods was assessed performing analyses that seek for monotonic or expected associations between, on the one hand, strain and displacement indices, and, on the other hand, clinical expertise expressed through a classification score referred to as the Wall Motion Score (WMS). This score, issued from the observation of the agreement between medical experts (a cardiologist and a radiologist), is used to classify each myocardial segment as follows: 1 = normal contraction, 2 = low moderate hypokinesia (reduced contraction), 3 = severe hypokinesia, 4 = akinesia (no contraction) and $5=$ dyskinesia (paradoxical motion during systole) [51], [52]. To assess clinical informational value of strain and displacement, a one way analysis of variance (ANOVA 1) was performed with each strain and displacement indices as dependent variable and WMS as a 4 classes explanatory variable. In case of significant ANOVA 1 (through Fisher statistics testing null hypothesis of no strain or displacement difference between the 4 WMS classes), a multiple comparisons procedure was performed using a Tukey honestly significant difference (THSD) test, to detail these differences, with a particular focus on monotonic patterns (e.g. strain increasing with WMS).

The clinical informational value of strain and displacement indices, and also of $a$ and $\varphi$ criteria, was performed towards WMS which was dichotomized between absence (WMS from 1 to 4 ) or presence $(\mathrm{WMS}=5)$ of a dyskinesia. To perform such analyses, we used a Wilcoxon test to compare $a$ and $\varphi$ criteria between dyskinetic and non dyskinetic myocardial sectors. A logistic regression model was also performed with dyskinesia as binary dependent variable and all aforementioned continuous indices as explanatory variables. A particular focus was placed on discrimination using the area under the ROC curve (AUROCC) estimated for each criterion, denoting the probability that two myocardial sectors range in the expected order (e.g. lower value for non dyskinetic myocardial motion). All statistical analyses were performed on the Statistical Analysis System (SAS v9.4 for Windows, SAS Institute Inc., Cary, NC, USA) with a double-sided type I error set at 0.05 .

\section{RESULTS}

\section{A. Validation with Simulated Data}

Figure 4 shows how parameters are recovered by the assimilation process DASS2 with respect to the respective ground truth values used for simulated image dataset \#1 (Fig. 1).

1) Influence of an occultation artefact: Displacement fields are estimated, on the one hand by using the assimilation method with the standard transport model (DASS1) and with the proposed dynamical model (DASS2), and on the other hand with the observation term alone (OBS) (Fig. 5b)for the four simulated DBAs. A three modality factor was considered for the pixels of the image sequence: $0=$ when DBA does not exist, $1=$ when located in the DBA region but DBA is not present ( 3 out of 4 images) and $2=$ when DBA is really applied ( 1 out of 4 images). Significant interaction was found between the methods and DBA $(p<0.0001)$. Similar DBA effects were found for OBS and DASS1, with mean errors $( \pm \mathrm{SE})$ as follows: $-0.05021 \pm 0.00031 \mathrm{~mm}$ (DBA 0), $-0.89922 \pm 0.02475 \mathrm{~mm}$ (DBA 1) and $-0.4073 \pm 0.00664 \mathrm{~mm}$ (DBA 2) for OBS, and $0.0452 \pm 0.00028 \mathrm{~mm}$ (DBA 0), $-0.76408 \pm 0.01982 \mathrm{~mm}$ (DBA 1) and $-0.3749 \pm 0.00598 \mathrm{~mm}$ (DBA 2) for DASS1. DBA exhibited a very different effect for DASS2 with much smaller errors, notably for DBA 1 and 2 that were not significantly different $(\mathrm{p}=0.3305)$ : $0.01162 \pm 0.00008 \mathrm{~mm}$ (DBA 0), $-0.07077 \pm 0.00097 \mathrm{~mm}$ (DBA 1) and $-0.08794 \pm 0.00211 \mathrm{~mm}$ (DBA 2).

2) Influence of noise: The means and standard deviations of errors for the three methods OBS, DASS1 and DASS2 with respect to the expected displacement field are plotted in Fig. 6 for four signal-tonoise ratios $(\infty, 20,10$ and 5$)$ for the four simulated deformations. Significant interaction was found between the methods and noise $(p<0.0001)$. Similar noise effects were found for OBS and DASS1, with mean errors $( \pm$ SE) as follows: $-0.07042 \pm 0.0004 \mathrm{~mm}$ $(\mathrm{SNR} \infty$ ), $-0.08083 \pm 0.00047 \mathrm{~mm}$ (SNR 20), $-0.09634 \pm 0.00052 \mathrm{~mm}$ (SNR 10) and $-0.1156 \pm 0.00064 \mathrm{~mm}$ (SNR 5) for OBS, and $-0.07077 \pm 0.00043 \mathrm{~mm} \quad(\mathrm{SNR} \infty), \quad-0.08469 \pm 0.00054 \mathrm{~mm} \quad$ (SNR 20), $-0.10417 \pm 0.00061 \mathrm{~mm}$ (SNR 10) and $-0.1254 \pm 0.00075 \mathrm{~mm}$ (SNR 5) for DASS1. Noise exhibited a very different effect for DASS2 with much smaller errors, notably for the first three levels of noise that were not significantly different $(p>0.05$ for all 6 paired comparisons): $-0.00698 \pm 0.00004 \mathrm{~mm}(\mathrm{SNR} \infty)$, $0.00707 \pm 0.00004 \mathrm{~mm}$ (SNR 20), $-0.00674 \pm 0.00004 \mathrm{~mm}$ (SNR 10) and $-0.00912 \pm 0.00006 \mathrm{~mm}$ (SNR 5).

\section{B. Application to Clinical Data}

Cardiac motion was estimated from real cine MRI using the 3 methods DASS1, DASS2 and OBS, and retrieved from tagged MRI images using inTag software. Figure 7 illustrates the typical displacement fields recovered for one frame of one patient with DASS2 and inTag.

Displacement fields were then spatially differentiated to give the strain tensor, transformed to the local myocardium coordinate system (radial/circumferential). Its components averaged over all of the 16 AHA divisions of the myocardium were compared with the ones 


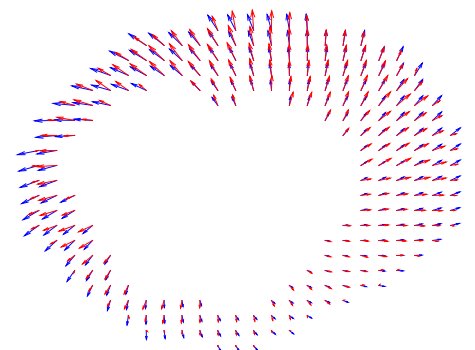

(a)

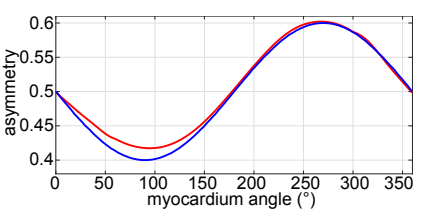

(b)

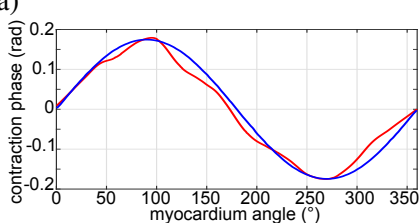

(c)
Fig. 4: Parameters estimated by DASS2 for the simulated image dataset \#1: displacement field $\boldsymbol{v}$ (a), angular variations of asymmetry $a$ (b) and contraction phase $\varphi$ (c). Estimated values in red superimposed with their respective ground truth in blue.

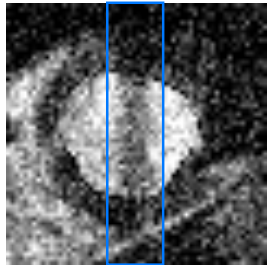

(a) (b)

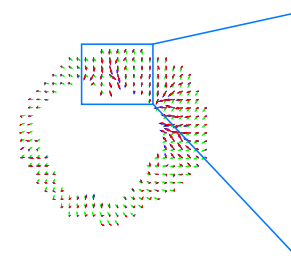

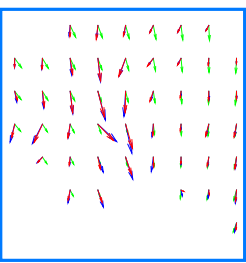

(c)
Fig. 5: (a) Simulated vertical dark band artefact with added Gaussian noise of $S N R=10$; (b) estimated displacement field with DASS2 (red) and OBS (green) superimposed with ground truth (blue); (c) displacement field zoomed over the artefact region.

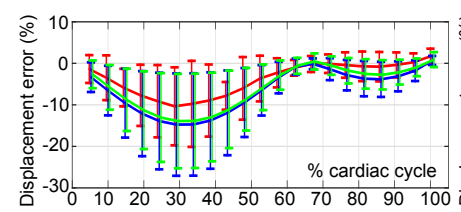

(a)

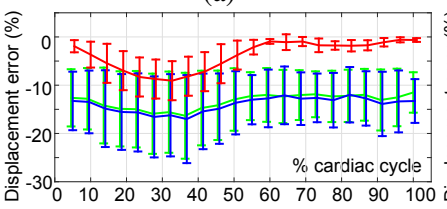

(c)

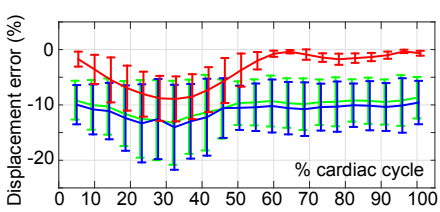

(b)

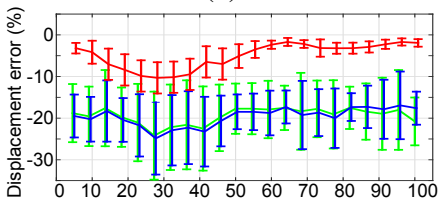

(d)
Fig. 6: Mean and standard deviation of the errors between ground truth and estimated displacements for the three methods OBS (green), DASS1 (blue) and DASS2 (red) for simulated image dataset \#1. Gaussian noise with different SNRs was added to the simulated sequence: $\infty$ (a), 20 (b), 10 (c) and 5 (d).

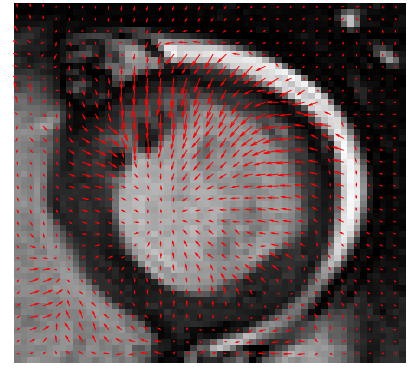

(a)

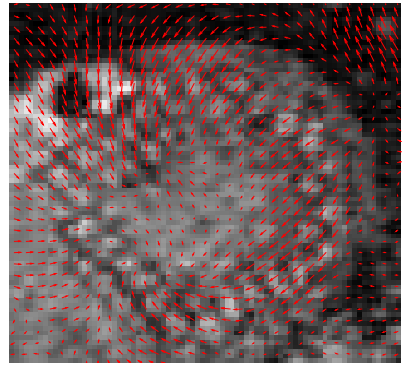

(b)
Fig. 7: Cine and tagged MRI in short-axis view with superimposed displacement fields at systole time estimated by DASS2 (a) and inTag (b) respectively.
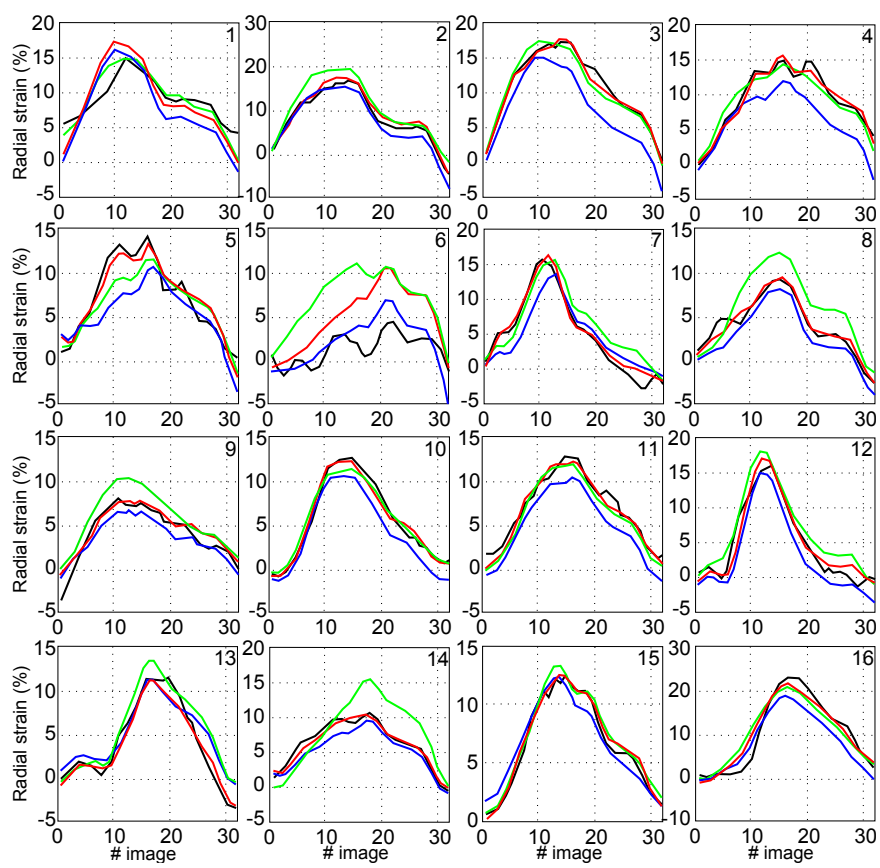

Fig. 8: Radial strain component (\%) estimated at AHA sectors 1 to 16 over one cardiac cycle for one patient of the database with inTag (black) from tagged MRI and with DASS2 (red), DASS1 (blue) and OBS (green) from cine MRI.

retrieved from tagged MRI by the inTag software. Figures 8 and 9 respectively give the temporal variations of radial and circumferential components of strain for one representative patient with the four methods.

1) Evaluation of the concordance of strain and displacement measures between inTag and DASS2 (Tab. I): Regarding displacement, the mean difference between measurements from inTag and DASS2 was $0.0291 \pm 0.0085 \mathrm{~mm}$ (mean $\pm \mathrm{SE}$ ) and was slightly significantly different from $0(p=0.0145)$. The ICC was 0.83 , reflecting a good agreement of displacement measures between methods. Regarding radial strain, the mean difference between measurements from inTag and DASS2 was $0.001 \pm 0.0023$ (mean \pm SE) and was not significantly different from 0 ( $p=0.6763)$. The ICC was 0.85 , reflecting a good agreement of radial strain measures between methods. Regarding circumferential strain, the mean difference between measurements from inTag and DASS2 was $0.0033 \pm 0.0015$ (mean \pm SE) and was not significantly different from $0(p=0.4992)$. The ICC was 0.89 , reflecting a very good agreement of circumferential strain measures 
TABLE I: RELIABILITY OF OBS, DASS 1 AND DASS2 VS. INTAG FOR THE CRITERIA OF DISPLACEMENT (DISP), RADIAL STRAIN (RS) AND CIRCUMFERENTIAL STRAIN (CS) WITH THE $p$-VALUE OF THE DISCORDANCE TEST (SIGNED RANK TEST) AND THE INTRACLASS CORRELATION COEFFICIENT (ICC).

\begin{tabular}{lllllll}
\hline Method & Criterion & $\mathrm{N}$ & Mean Difference (SE) & Median Difference [IQR] & $p$-value & ICC \\
\hline OBS & Disp $(m m)$ & 718 & $0.0372(0.0097)$ & $0.0212[-0.135 ; 0.2058]$ & 0.0016 & 0.8483 \\
& RS & 718 & $0.0001(0.0025)$ & $-0.0042[-0.0462 ; 0.0397]$ & 0.4204 & 0.8729 \\
& CS & 718 & $0.0047(0.0016)$ & $-0.0035[-0.0261 ; 0.0271]$ & 0.9650 & 0.8943 \\
DASS1 & Disp $(m m)$ & 718 & $-0.0165(0.0098)$ & $-0.0525[-0.1667 ; 0.1264]$ & 0.0009 & 0.5988 \\
& RS & 718 & $-0.0172(0.0016)$ & $-0.0144[-0.0423 ; 0.0091]$ & $9.7921 \mathrm{E}-25$ & 0.4740 \\
& CS & 718 & $-0.0141(0.0018)$ & $-0.012[-0.0406 ; 0.015]$ & $5.9564 \mathrm{E}-14$ & 0.5725 \\
DASS2 & Disp $(m m)$ & 718 & $0.0291(0.0085)$ & $0.0097[-0.1321 ; 0.1571]$ & 0.0145 & 0.8328 \\
& RS & 718 & $0.001(0.0023)$ & $-0.0024[-0.0404 ; 0.0353]$ & 0.6763 & 0.8548 \\
& CS & 718 & $0.0033(0.0015)$ & $-0.0055[-0.0238 ; 0.0223]$ & 0.4992 & 0.8878 \\
\hline
\end{tabular}

TABLE II: MUlTIPLE COMPARISONS USING TUKEY GROUPING OF DiSPLACEMENTS IN $m m$ AS ESTIMATED BY INTAG (1), OBS (2), DASS1 (3) AND DASS2 (4) WITH RESPECT TO WMS. MEANS SHARING THE SAME LETTER ARE NOT SIGNIFICANTLY DIFFERENT.

\begin{tabular}{llllll}
\hline WMS & N & Mean & SD & THSD \\
\hline 1 & 461 & 1.0368 & 0.4299 & A & \\
2 & 56 & 0.9135 & 0.4116 & A & B \\
3 & 101 & 0.8512 & 0.4455 & C & B \\
4 & 90 & 0.7427 & 0.3707 & C & \\
\hline \multicolumn{5}{c}{$(1)$}
\end{tabular}

\begin{tabular}{llllll}
\hline WMS & N & Mean & SD & THSD \\
\hline 1 & 461 & 1.1573 & 0.5524 & A & \\
2 & 56 & 0.8887 & 0.3832 & & B \\
3 & 101 & 0.8466 & 0.4578 & & B \\
4 & 90 & 0.6347 & 0.3559 & C & \\
\hline
\end{tabular}

\begin{tabular}{llllll}
\hline WMS & N & Mean & SD & \multicolumn{2}{l}{ THSD } \\
\hline 1 & 461 & 0.6805 & 0.3476 & A & \\
2 & 56 & 0.6651 & 0.2561 & A & B \\
3 & 101 & 0.5558 & 0.2562 & & B \\
4 & 90 & 0.4357 & 0.2567 & C & \\
\hline
\end{tabular}

(3)

\begin{tabular}{lllllr}
\hline WMS & N & Mean & SD & THSD \\
\hline 1 & 461 & 0.9646 & 0.4678 & A & \\
2 & 56 & 0.7546 & 0.3611 & & B \\
3 & 101 & 0.7184 & 0.3775 & & B \\
4 & 90 & 0.5459 & 0.2973 & C & \\
\hline
\end{tabular}

(4)

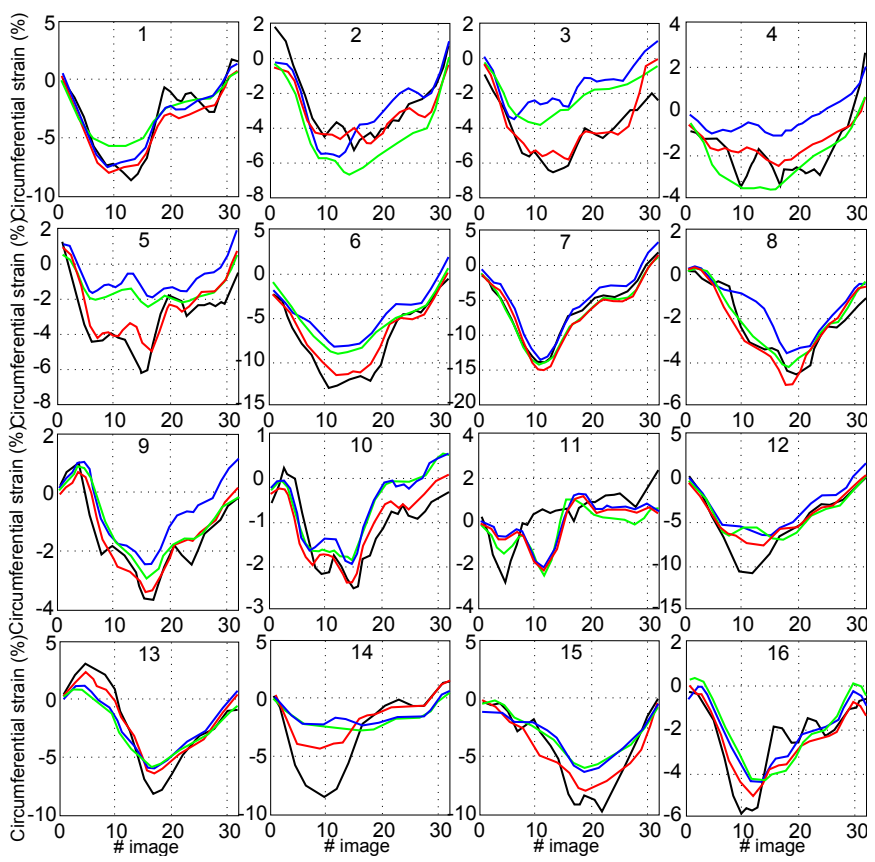

Fig. 9: Circumferential strain component (\%) estimated at AHA sectors 1 to 16 over one cardiac cycle for one patient of the database with inTag (black) from tagged MRI, and with DASS2 (red), DASS1 (blue) and OBS (green) from cine MRI.

between methods. Results for OBS were comparable to DASS2 ones with a slightly lower offset with regard to inTag, while for DASS1 discordance is significant for all criteria with a trend to underestimation and poor ICC values. The corresponding Bland and Altman plots are displayed in Fig. 10.
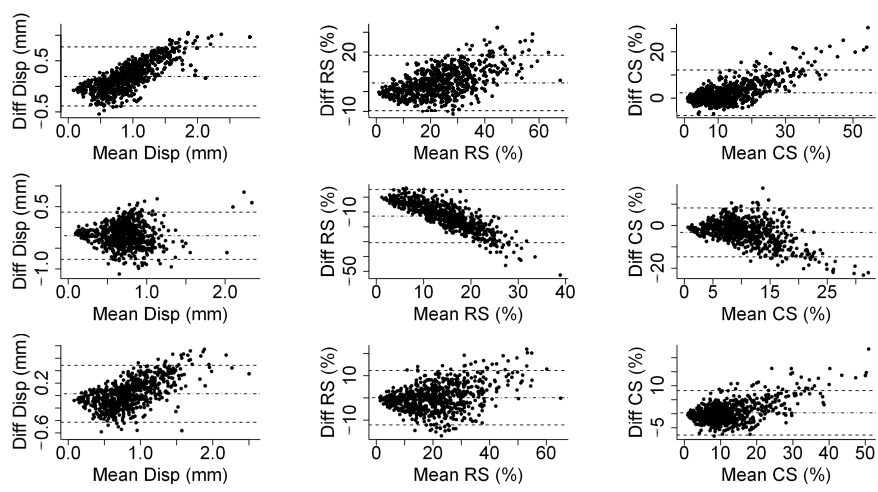

Fig. 10: Bland and Altman plots of displacement, radial and circumferential strain components for OBS (top), DASS1 (middle) and DASS2 (bottom) vs. inTag. The difference to inTag is plotted against the mean.

2) Evaluation of the clinical informational value of strain and displacement indices regarding the Wall Motion Score (WMS) from 1 (normal contraction) to 4 (akinesia): Regardless of the method used, inTag or DASS2, both strain and displacement were significantly different regarding the 4 classes of WMS (all $p$-values lower than 0.0001). Furthermore, whatever the method, displacement, circumferential and radial strain ranged in descending order from WMS 1 to WMS 4 (Tab. II and III). Regarding displacement, the multiple comparisons procedure using THSD showed that the couples of classes not significantly different were 1-2 and 2-3 for inTag, and only 2-3 for DASS2 (Tab. II). Regarding circumferential strain, the multiple comparisons procedure using THSD showed that the couples of classes not significantly different were 1-2 and 2-3 for inTag, while classes 1, 2 and 3 were undistinguishable for DASS2 (Tab. III). Regarding radial strain, the multiple comparisons procedure using 
TABLE III: MUltiple COMPARISONS USING TUKEy GROUPing OF STRAin AS ESTIMATED By INTAG (1), OBS (2), DASS1 (3) AND DASS2 (4) WITH RESPECT TO WMS. (A) AND (B) ARE THEIR RESPECTIVE RADIAL AND CIRCUMFERENTIAL COMPONENTS. MEANS SHARING THE SAME LETTER ARE NOT SIGNIFICANTLY DIFFERENT.

\begin{tabular}{lllllr}
\hline WMS & N & Mean & SD & THSD \\
\hline 2 & 56 & 0.182 & 0.0841 & A & \\
1 & 461 & 0.1725 & 0.0867 & A & \\
3 & 101 & 0.1648 & 0.0868 & A & \\
4 & 90 & 0.1127 & 0.0573 & & B
\end{tabular}

(1.a)

\begin{tabular}{llllll}
\hline WMS & N & Mean & SD & \multicolumn{2}{l}{ THSD } \\
\hline 1 & 461 & 0.0885 & 0.0307 & A & \\
2 & 56 & 0.0826 & 0.0281 & A & \\
3 & 101 & 0.078 & 0.0362 & A & \\
4 & 90 & 0.0619 & 0.0358 & & B
\end{tabular}

(3.a)

\begin{tabular}{llllll}
\hline WMS & N & Mean & SD & THSD \\
\hline 1 & 461 & 0.1344 & 0.0599 & A & \\
2 & 56 & 0.1284 & 0.0589 & A & B \\
3 & 101 & 0.11 & 0.0529 & & B \\
4 & 90 & 0.0782 & 0.0486 & C &
\end{tabular}

(1.b)

\begin{tabular}{llllll}
\hline WMS & N & Mean & SD & THSD \\
\hline 1 & 461 & 0.0999 & 0.0435 & A & \\
2 & 56 & 0.0948 & 0.0379 & A & \\
3 & 101 & 0.077 & 0.0414 & & B \\
4 & 90 & 0.0527 & 0.035 & C & \\
\hline
\end{tabular}
(3.b)

\begin{tabular}{lllllr}
\hline WMS & N & Mean & SD & THSD \\
\hline 1 & 461 & 0.2947 & 0.1356 & A & \\
2 & 56 & 0.2287 & 0.0962 & & B \\
3 & 101 & 0.1997 & 0.1327 & & B \\
4 & 90 & 0.1306 & 0.0864 & C & \\
\hline
\end{tabular}
(2.a)

\begin{tabular}{lllllr}
\hline WMS & N & Mean & SD & THSD \\
\hline 1 & 461 & 0.2471 & 0.121 & A & \\
2 & 56 & 0.1798 & 0.0814 & & B \\
3 & 101 & 0.1665 & 0.1159 & & B \\
4 & 90 & 0.1067 & 0.0691 & C & \\
\hline
\end{tabular}
(4.a)

\begin{tabular}{lllllr}
\hline WMS & N & Mean & SD & THSD \\
\hline 1 & 461 & 0.16 & 0.1123 & A & \\
2 & 56 & 0.1431 & 0.0908 & A & \\
3 & 101 & 0.131 & 0.1053 & A & \\
4 & 90 & 0.0886 & 0.0682 & & B
\end{tabular}
(2.b)

\begin{tabular}{lllllr}
\hline WMS & $\mathrm{N}$ & Mean & SD & THSD \\
\hline 1 & 461 & 0.1385 & 0.0989 & A & \\
2 & 56 & 0.1241 & 0.0804 & A & \\
3 & 101 & 0.1124 & 0.089 & A & \\
4 & 90 & 0.077 & 0.0576 & & B \\
\hline
\end{tabular}
(4.b)

TABLE IV: COMPARISONS OF DISPLACEMENT (DISP), RADIAL STRAIN (RS) AND CIRCUMFERENTIAL STRAIN (CS) FOR INTAG, OBS, DASS1 AND DASS2 AND $a$ AND $\varphi$ FOR DASS2 BETWEEN DYSKINETIC AND NON DYSKINETIC MYOCARDIAL AHA DIVISIONS PERFORMING A WILCOXON TEST AND ITS CORRESPONDING AURROC.

\begin{tabular}{|c|c|c|c|c|c|c|c|}
\hline \multirow[t]{2}{*}{ Method } & \multirow[t]{2}{*}{ Criterion } & \multicolumn{2}{|c|}{ WMS $\leq 4(\mathrm{~N}=708)$} & \multicolumn{2}{|c|}{ WMS $=5(\mathrm{~N}=10)$} & \multirow{2}{*}{$\begin{array}{l}p \text {-value } \\
\text { (Wilcoxon) }\end{array}$} & \multirow[t]{2}{*}{ AUROCC } \\
\hline & & Mean \pm SE & Median [IQR] & Mean \pm SE & Median [IQR] & & \\
\hline \multirow[t]{3}{*}{ inTag } & Disp $(\mathrm{mm})$ & $0.9632 \pm 0.0164$ & $0.8939[0.6303 ; 1.2563]$ & $0.722 \pm 0.1183$ & $0.6649[0.3587 ; 0.8243]$ & 0.0662 & 0.677 \\
\hline & $\mathrm{RS}$ & $0.1645 \pm 0.0032$ & $0.1466[0.1022 ; 0.2156]$ & $0.1148 \pm 0.0168$ & $0.0948[0.0784 ; 0.1598]$ & 0.0565 & 0.674 \\
\hline & CS & $0.1233 \pm 0.0023$ & $0.1151[0.0783 ; 0.1647]$ & $0.091 \pm 0.0159$ & $0.0912[0.0587 ; 0.135]$ & 0.1203 & 0.657 \\
\hline \multirow[t]{3}{*}{ OBS } & $\operatorname{Disp}(m m)$ & $1.0253 \pm 0.0203$ & $0.9402[0.6248 ; 1.347]$ & $0.886 \pm 0.1421$ & $0.8566[0.5949 ; 1.0686]$ & 0.4684 & 0.559 \\
\hline & RS & $0.2551 \pm 0.0053$ & $0.2418[0.151 ; 0.3406]$ & $0.1987 \pm 0.0373$ & $0.1577[0.1285 ; 0.2941]$ & 0.1823 & 0.627 \\
\hline & $\mathrm{CS}$ & $0.1455 \pm 0.004$ & $0.1157[0.0715 ; 0.1903]$ & $0.1124 \pm 0.0158$ & $0.1112[0.0906 ; 0.1449]$ & 0.6375 & 0.533 \\
\hline \multirow[t]{3}{*}{ DASS1 } & Disp & $0.6304 \pm 0.0124$ & $0.6102[0.4301 ; 0.8038]$ & $0.5552 \pm 0.1032$ & $0.458[0.2858 ; 0.7211]$ & 0.3772 & 0.588 \\
\hline & RS & $0.0831 \pm 0.0012$ & $0.0822[0.0608 ; 0.1032]$ & $0.0766 \pm 0.013$ & $0.0716[0.0446 ; 0.1008]$ & 0.4637 & 0.569 \\
\hline & $\mathrm{CS}$ & $0.0902 \pm 0.0017$ & $0.0899[0.0586 ; 0.1197]$ & $0.0636 \pm 0.0107$ & $0.0615[0.0338 ; 0.0759]$ & 0.0542 & 0.695 \\
\hline \multirow[t]{5}{*}{ DASS2 } & Disp $(m m)$ & $0.8596 \pm 0.0171$ & $0.7822[0.5282 ; 1.1296]$ & $0.7552 \pm 0.1204$ & $0.7199[0.5072 ; 0.9114]$ & 0.55 & 0.557 \\
\hline & RS & $0.2123 \pm 0.0046$ & $0.2006[0.122 ; 0.2863]$ & $0.1598 \pm 0.035$ & $0.1259[0.0666 ; 0.2514]$ & 0.1509 & 0.64 \\
\hline & CS & $0.1258 \pm 0.0035$ & $0.0994[0.0609 ; 0.1648]$ & $0.0996 \pm 0.014$ & $0.098[0.0794 ; 0.1351]$ & 0.7437 & 0.511 \\
\hline & $a$ & $0.8415 \pm 0.0086$ & $0.9388[0.6959 ; 1.0028]$ & $0.6889 \pm 0.0605$ & $0.6458[0.534 ; 0.7536]$ & 0.0369 & 0.697 \\
\hline & $\varphi(\mathrm{rad})$ & $1.0104 \pm 0.0184$ & $1.108[0.7093 ; 1.3108]$ & $0.6309 \pm 0.1418$ & $0.5867[0.2885 ; 0.835]$ & 0.0122 & 0.733 \\
\hline
\end{tabular}

THSD showed that the couples of classes not significantly different were only 2 and 3 for both inTag and DASS2 (Tab. III).

3) Evaluation of the clinical informational value of strain and displacement indices, $a$ and $\varphi$ criteria regarding the diagnosis of dyskinesia (dichotomizing WMS less or equal to 4 vs. 5) (Table $I V)$ : Regarding displacement, the median value as measured by inTag was $0.89 \mathrm{~mm}[\operatorname{IQR}(\mathrm{mm})=1.25-1.63]$ for non dyskinetic versus $0.66 \mathrm{~mm}[\operatorname{IQR}(\mathrm{mm})=0.82-0.36]$ for dyskinetic sectors $(p=0.0662)$, while for DASS2 the median value was $0.78 \mathrm{~mm}$ [IQR $(\mathrm{mm})=1.13$ $0.53]$ for non dyskinetic versus $0.72 \mathrm{~mm}$ [IQR $(\mathrm{mm})=0.91-0.51]$ for diskinetic sectors $(p=0.55)$. The discriminant power of displacement measured by inTag and DASS2 regarding dyskinesia, as assessed by AUROCC, was 0.677 and 0.557 , respectively. Regarding radial strain, the median value as measured by inTag was 0.15 [IQR $=0.22-$ 0.11] for non dyskinetic versus 0.095 [IQR=0.16-0.078] for dyskinetic sectors $(p=0.0565)$, while for DASS2 the median value was
$0.20[\mathrm{IQR}=0.29-0.12]$ for non dyskinetic versus 0.13 [IQR $=0.25$ $0.067]$ for dyskinetic sectors $(p=0.1509)$. The discriminant power of radial strain measured by inTag and DASS2 regarding dyskinesia, as assessed by AUROCC, was 0.674 and 0.64, respectively. Regarding circumferential strain, the median value as measured by inTag was 0.11 [IQR=0.15-0.078] for non diskinetic versus 0.095 [IQR=0.13$0.059]$ for diskinetic sectors $(p=0.1203)$, while for DASS 2 the median value was 0.099 [IQR $=0.16-0.061]$ for non dyskinetic versus 0.099 $[\mathrm{IQR}=0.14-0.079]$ for dyskinetic sectors $(p=0.7437)$. The discriminant power of circumferential strain measured by inTag and DASS2 regarding dyskinesia, as assessed by AUROCC, was 0.657 and 0.511, respectively. Results for OBS were comparable to DASS2 ones, while they were less good for DASS1. Regarding $a$, the median value was $0.94[\mathrm{IQR}=1.00-0.70]$ for non dyskinetic versus 0.65 [IQR $=0.75$ 0.53 ] for dyskinetic sectors $(p=0.0369)$. Regarding $\varphi$, the median value was $1.11 \mathrm{rad}[\mathrm{IQR}(\mathrm{rad})=1.31-0.71]$ for non dyskinetic versus 
$0.59 \mathrm{rad}[\mathrm{IQR}(\mathrm{rad})=0.84-0.29]$ for dyskinetic sectors $(p=0.0122)$. The discriminant power of $a$ and $\varphi$ regarding dyskinesia, as assessed by AUROCC, was 0.695 and 0.733 , respectively.

\section{DISCUSSION}

Figure $4 \mathrm{a}$ shows that the displacement field is almost perfectly estimated by DASS2 with respect to the ground truth field, and we also get very good spatial distribution of the contraction parameters, i.e. contraction phase $\varphi$ and factor of asymmetry a compared to simulations (Fig. 4b and 4c). Angular variations of the parameters are properly estimated even in presence of high noise $(S N R=10)$, which is a promising sign for detecting local asynchrony of contraction in real data.

Figure 6 shows that the error is roughly proportional to the motion amplitude, and in presence of noise, systematically smaller for DASS2 than for DASS1 or OBS. The difference increases with the amount of noise: error remains rather stable for DASS2 as a result of the proposed dynamical model that properly smooths temporal variations of the state variables separately for systole and diastole. Variability of the estimation is also greatly improved. The smoothing by the continuous transport model of DASS1 tends to mix contraction and dilation phases, and therefore to underestimate displacement amplitude, making the error higher than the one of OBS at diastole.

The simulation of the dark band artifact confirms the robustness of the dynamical model of DASS2 (Fig. 5). Indeed, for the observation operator, the dark band loss from one frame to the next is equivalent to an horizontal inward flow. Zoom over the dark band region (Fig. 5b) shows that the proposed piecewise transport model compensates the result when the observation operator fails due to temporary occultation or loss in signal. The statistical analysis reveals that errors are about ten times lower for DASS2 and that they are even higher for both OBS and DASS1 for locations inside the DBA region but without effective attenuation.

For real MRI datasets, figure 7 shows that the motion fields estimated by inTag and DASS2 are almost identical. The differences come from the following key points: inTag provides very smooth fields with non null circumferential component and a radial component sometimes underestimated due to the lack of tagging patterns within the myocardium thickness; for DASS2, motion is null outside myocardium except for the blood pool where flow turbulences hold, and non radial motion only happens where endocardium is non smooth (trabeculae or pillars).

Figures 8 and 9 illustrate representative trends for temporal profiles of strain averaged over AHA sectors. There is visually a good match between inTag and DASS2 profiles for most of the sectors, with amplitudes that comply with expected values described in the literature of speckle tracking. For some sectors however, radial component is underestimated by inTag, while for others the circumferential component is underestimated by DASS2. Profiles of OBS is usually higher than the other profiles, because optical flow constraint is influenced by variations in image intensity, either due to noise or to artifacts. In particular, the fact that through slice motion was not compensated is likely to cause signal variations at myocardial walls, and create artefactual inplane motion, possibly filtered by the transport model of DASS2. Again, DASS1 is subject to underestimate displacement and therefore corresponding strain profiles are lower.

Table I and the corresponding Bland and Altman plots (Fig. 10) emphasize these observations. Indeed, DASS1 exhibits poor agreement and systematic bias as compared with inTag for all the criteria, while OBS and DASS2, even if a slight overestimation is noticed for displacement, show very good agreement for all criteria.

The clinical informational value of displacement measured by the four methods is shown in Table II. We see that displacement ranges in the expected order for all methods, and that DASS2 and OBS better discriminate WMS than DASS1 and inTag, the latter being almost unable to distinguish between the four clinical classes. As shown in table III, we found similar results for radial strain component: DASS2 and OBS significantly discriminate extreme scores 1 and 4 from each other and relative to intermediate scores 2 and 3, the latter being non significantly different. On the contrary, circumferential strain component poorly distinguishes WMS classes for OBS and DASS2 as compared with inTag or even DASS1.

Despite WMS being a subjective measure, it integrates expert knowledge at two levels: first, sectors are not scored independently from others in a given slice, but relatively to them; second, the cardiologist or the radiologist implicitly accounts for the characteristics of the patient such as age or gender for instance. On contrary, automatic quantification methods give absolute strain values and do not take into account patient variability. It is noticeable that this variability and the intrinsic one of strain estimation do not hide the discrimination between WMS classes. DASS2 seems more trustful to discriminate between WMS with radial component than inTag, while for the circumferential component, inTag is better than DASS2. Indeed, the variability of the radial measurement with inTag comes from the fact that there might be too few tags to track in the thickness for some sectors. For the circumferential measurement with DASS2, there might be no angular gradient features to track when myocardial walls are too smooth. Informational value of DASS2 and OBS are similar for good cine MRI acquisitions, but discrepancies could occur in presence of noise or artifacts as illustrated by the simulation study. The weak informational value of DASS1 can be explained by the smoothing performed by the continuous evolution model.

Table IV gives the informational value of the criteria regarding dyskinesia. It shows that only $a$ and $\varphi$ are significantly different between dyskinetic and non dyskinetic patients, with quite good AUROCC. This result suggests that these two criteria should be investigated on a larger number of patients before they could be used as objective markers of dyskinesia.

One of the present limitation of both inTag and cine MRI based methods such as DASS2 is that in-plane motion is influenced by through-plane motion due to heart contraction. Only apparent motion is provided; estimating pure strain components would require full 3D acquisitions instead of thick and spaced slices. Furthermore, it would be necessary to know the exact limits of the myocardial walls to get more accurate measurements in the sectors. For now we use an approximate segmentation of myocardium. This is legitimate in the variational approach developed here, as the regularization term effectively cancels the displacement fields outside of the myocardium, especially in the cavity.

\section{Conclusion and Future Prospects}

This paper reports a method for estimating cardiac contractility parameters in a formalism of variational data assimilation that combines the image observations with a dynamic evolution model of the heart. The method was applied to a synthetic data set where the field of real movement is known and clinical data of cine MRI sequences from 47 patients presenting areas of impaired kinetics. The results were compared with those obtained with inTag tissue tagging tracking software. The automatic contractility estimation was also cross-compared against the cardiological and radiological expertise through semi-quantitative contraction score.

The proposed method yields promising results for both motion and dyskinesia index estimation. For now, the displacement and strain components in radial direction demonstrate the best performance, but it is interesting to note that the circumferential components are only slightly recovered due to shape irregularities at the endocardium wall 
caused by the presence of papillary muscles or trabeculae and are able to discriminate the kinetic scores. They could be improved by including directional constraints in the model.

As future prospects, a wall deformation model will be added as part of the state vector to solve segmentation and motion estimation as a joint problem. DASS2 is being extended to 3D dynamic imaging modalities such as echocardiography and Single-Photon Emission Computed Tomography (SPECT), giving promising results for the recovery of all strain components.

The final limit of any strain estimation method is the variability in strain-normal values that are known to be dependent on gender and age among others. Normalization of strain indices in terms of nonlinear relationships with patient characteristics is important as a field of further research to enable diagnosis of infraclinical functional abnormalities in the future.

\section{APPENDIX}

\section{A - OPTIMIZATION OF THE VARIATIONAL ASSIMILATION PROBLEM}

Using algorithm 1 requires to derive linear-tangent and adjoints of models $\mathbb{M}$ and $\mathbb{H}$. Their computation is presented in this appendix.

Dynamical model: Given the definition of the Gâteaux derivative in (5), we get :

$$
\begin{gathered}
\frac{\partial \mathbb{M}}{\partial \mathcal{X}}(\delta \mathcal{X})=\lim _{\beta \rightarrow 0}\left\{\frac{[\operatorname{sgn}(\sin (\omega t+\varphi+\beta \delta \varphi)+a+\beta \delta a)]}{\beta}\right. \\
\left.\frac{(\mathbf{v}+\beta \delta \mathbf{v})^{\mathrm{T}} \nabla(\mathcal{X}+\beta \delta \mathcal{X})-[\operatorname{sgn}(\sin (\omega t+\varphi)+a)] \mathbf{v}^{\mathrm{T}} \nabla \mathcal{X}}{\beta}\right\} .
\end{gathered}
$$

In practice, the function $\operatorname{sgn}(x)$ can be approximated in the continuous domain by $\tanh (k x)$ with $k>1$. After Taylor expansion $\tanh [k(x+\beta \delta x)]=\tanh (k x)+k \beta \delta x\left(1-\tanh ^{2}(k x)\right)$ and $\sin (\omega t+\varphi+\beta \delta \varphi)=\sin (\omega t+\varphi)+\beta \delta \varphi \cos (\omega t+\varphi)$ and putting together terms in $\beta, \beta^{2}, \ldots$, we get to the following equation:

$$
\frac{\partial \mathbb{M}}{\partial \mathcal{X}}(\delta \mathcal{X})=A_{1}\left(\delta \mathbf{v}^{\mathrm{T}} \nabla \mathcal{X}+\mathbf{v}^{\mathrm{T}} \nabla \delta \mathcal{X}\right)+\left(A_{k} \delta \varphi+k A_{2} \delta a\right) \mathbf{v}^{\mathrm{T}} \nabla \mathcal{X}
$$

with $A_{1}=\tanh [k(\sin (\omega t+\varphi)+a)], A_{2}=1-\tanh ^{2}[k(\sin (\omega t+$ $\varphi)+a)]$ and $A_{k}=k A_{2} \cos (\omega t+\varphi)$. The global linear tangent model is then: $\frac{\partial \delta \mathcal{X}}{\partial t}+\frac{\partial \mathbb{M}}{\partial \mathcal{X}}(\delta \mathcal{X})=\nu_{m}$. The adjoint model operator will be given in the next section.

Observation model: Following the same principle, the linear tangent model of operator $\mathbb{H}_{\mathbf{v}}$ and $\mathbb{H}_{\varphi, a}$ read:

$$
\begin{aligned}
\partial \mathbb{H}_{\mathbf{v}} & =\left(\frac{\partial \mathbb{H}_{\mathbf{v}}}{\partial \mathcal{X}}\right) \delta \mathbf{v}=\nabla I^{\mathrm{T}} \\
\partial \mathbb{H}_{\varphi} & =\left(\frac{\partial \mathbb{H}_{\varphi, a}}{\partial \mathcal{X}}\right) \delta \varphi=-\frac{\Lambda-\widehat{\Lambda}}{\sigma_{\Lambda}} \cos (\omega t+\varphi) \\
\partial \mathbb{H}_{a} & =\left(\frac{\partial \mathbb{H}_{\varphi, a}}{\partial \mathcal{X}}\right) \delta a=-\frac{\Lambda-\widehat{\Lambda}}{\sigma_{\Lambda}}
\end{aligned}
$$

\section{Adjoint operators}

Let us recall that the adjoint $\mathbb{A}^{*}$ of a linear operator $\mathbb{A}$ on a space $\mathcal{D}$ is such that:

$$
\forall x_{1}, x_{2} \in \mathcal{D},<\mathbb{A} x_{1}, x_{2}>=<x_{1}, \mathbb{A}^{*} x_{2}>.
$$

In the following we derive their expressions for the dynamical and observation models respectively.

Dynamical model: Recalling that the system state $\mathcal{X}$ is composed of four variables : $\mathcal{X}=(u(\mathbf{x}, t), v(\mathbf{x}, t), \varphi(\mathbf{x})$, we first derive the linear tangent operators of $\mathbb{M}$ with respect to each of these variables (with $A_{1}$ and $A_{2}$ develop above):

$$
\begin{array}{r}
\left(\frac{\partial \mathbb{M}}{\partial \mathcal{X}}\right) \delta \mathcal{X}=\left(\frac{\partial \mathbb{M}_{1}}{\partial \mathcal{X}}(\delta \mathcal{X}), \frac{\partial \mathbb{M}_{2}}{\partial \mathcal{X}}(\delta \mathcal{X}), \frac{\partial \mathbb{M}_{3}}{\partial \mathcal{X}}(\delta \mathcal{X}), \frac{\partial \mathbb{M}_{4}}{\partial \mathcal{X}}(\delta \mathcal{X})\right)^{\mathrm{T}} \\
=\left(\begin{array}{c}
A_{1}\left(u \delta u_{x}+v \delta u_{y}+u_{x} \delta u+u_{y} \delta v\right)+\left(u u_{x}+v u_{y}\right)\left(A_{k} \delta \varphi+k A_{2} \delta a\right) \\
A_{1}\left(u \delta v_{x}+v \delta v_{y}+v_{x} \delta u+v_{y} \delta v\right)+\left(u v_{x}+v v_{y}\right)\left(A_{k} \delta \varphi+k A_{2} \delta a\right) \\
A_{1}\left(u \delta \varphi_{x}+v \delta \varphi_{y}+\varphi_{x} \delta u+\varphi_{y} \delta v\right)+\left(u \varphi_{x}+v \varphi_{y}\right)\left(A_{k} \delta \varphi+k A_{2} \delta a\right) \\
\left.A_{1}\left(u \delta a_{x}+v \delta a_{y}+a_{x} \delta u+a_{y} \delta v\right)+\left(u a_{x}+v a_{y}\right)\left(A_{k} \delta \varphi+k A_{2} \delta a\right)\right)
\end{array}\right)
\end{array}
$$

The computation of adjoint operator $\left(\frac{\partial \mathbb{M}}{\partial \mathcal{X}}\right)^{*}$ can be done using integration by parts :

$$
\begin{aligned}
& \int \frac{\partial \mathbb{M}}{\partial \mathcal{X}}(\boldsymbol{\psi})^{\mathrm{T}} \boldsymbol{\lambda} d \mathbf{x} d t \\
= & \int\left(\frac{\partial \mathbb{M}_{1}}{\partial \mathcal{X}}(\boldsymbol{\psi}) \lambda_{1}+\frac{\partial \mathbb{M}_{2}}{\partial \mathcal{X}}(\boldsymbol{\psi}) \lambda_{2}+\frac{\partial \mathbb{M}_{3}}{\partial \mathcal{X}}(\boldsymbol{\psi}) \lambda_{3}+\frac{\partial \mathbb{M}_{4}}{\partial \mathcal{X}}(\boldsymbol{\psi}) \lambda_{4}\right) d \mathbf{x} d t
\end{aligned}
$$

$$
\begin{aligned}
= & \int\left\{A_{1}\left[\left(-u\left(\lambda_{1}\right)_{x}-v\left(\lambda_{1}\right)_{y}-v_{y} \lambda_{1}\right) \psi_{1}+u_{y} \lambda_{1} \psi_{2}\right]+\lambda_{1}\left(A_{k} \psi_{3}\right.\right. \\
& \left.+k A_{2} \psi_{4}\right) \mathbf{v}^{\mathrm{T}} \nabla u+A_{1}\left[\left(-u\left(\lambda_{2}\right)_{x}-v\left(\lambda_{2}\right)_{y}-u_{x} \lambda_{2}\right) \psi_{2}+v_{x} \lambda_{2} \psi_{1}\right] \\
& +\lambda_{2}\left(A_{k} \psi_{3}+k A_{2} \psi_{4}\right) \mathbf{v}^{\mathrm{T}} \nabla v+A_{1}\left[\left(-u\left(\lambda_{3}\right)_{x}-v\left(\lambda_{3}\right)_{y}-u_{x} \lambda_{3}\right.\right. \\
& \left.\left.-v_{y} \lambda_{3}\right) \psi_{3}+\varphi_{x} \lambda_{3} \psi_{1}+\varphi_{y} \lambda_{3} \psi_{2}\right]+\lambda_{3}\left(A_{k} \psi_{3}+k A_{2} \psi_{4}\right) \mathbf{v}^{\mathrm{T}} \nabla \varphi \\
& +A_{1}\left[\left(-u\left(\lambda_{4}\right)_{x}-v\left(\lambda_{4}\right)_{y}-u_{x} \lambda_{4}-v_{y} \lambda_{4}\right) \psi_{4}+a_{x} \lambda_{4} \psi_{1}+a_{y} \lambda_{4} \psi_{2}\right] \\
& \left.+\lambda_{4}\left(A_{k} \psi_{3}+k A_{2} \psi_{4}\right) \mathbf{v}^{\mathrm{T}} \nabla a\right\} \mathbf{x} d t \\
= & \int\left(\left(\frac{\partial \mathbb{M}_{1}}{\partial \mathcal{X}}\right)^{*} \psi_{1}+\left(\frac{\partial \mathbb{M}_{2}}{\partial \mathcal{X}}\right)^{*} \psi_{2}+\left(\frac{\partial \mathbb{M}_{3}}{\partial \mathcal{X}}\right)^{*} \psi_{3}+\left(\frac{\partial \mathbb{M}_{4}}{\partial \mathcal{X}}\right)^{*} \psi_{4}\right) d \mathbf{x} d t \\
= & \int\left(\frac{\partial \mathbb{M}}{\partial \mathcal{X}}\right)^{*}(\boldsymbol{\lambda})^{\mathrm{T}} \boldsymbol{\psi} d \mathbf{x} d t .
\end{aligned}
$$

Given previous relations, the adjoint operator is given by the following vector as:

$$
\left(\frac{\partial \mathbb{M}}{\partial \mathcal{X}}\right)^{*}(\boldsymbol{\lambda})=\left(\begin{array}{c}
A_{1}\left[-u\left(\lambda_{1}\right)_{x}-v\left(\lambda_{1}\right)_{y}-v_{y} \lambda_{1}+v_{x} \lambda_{2}+\varphi_{x} \lambda_{3}+a_{x} \lambda_{4}\right] \\
A_{1}\left[-u\left(\lambda_{2}\right)_{x}-v\left(\lambda_{2}\right)_{y}-u_{x} \lambda_{2}+u_{y} \lambda_{1}+\varphi_{y} \lambda_{3}+a_{y} \lambda_{4}\right] \\
A_{1}\left[-u\left(\lambda_{3}\right)_{x}-v\left(\lambda_{3}\right)_{y}-u_{x} \lambda_{3}-v_{y} \lambda_{3}\right] \\
+A_{k} \mathbf{v}^{\mathrm{T}}\left[\lambda_{1} \nabla u+\lambda_{2} \nabla v+\lambda_{3} \nabla \varphi+\lambda_{4} \nabla a\right] \\
A_{1}\left[-u\left(\lambda_{4}\right)_{x}-v\left(\lambda_{4}\right)_{y}-u_{x} \lambda_{4}-v_{y} \lambda_{4}\right] \\
+k A_{2} \mathbf{v}^{\mathbf{T}}\left[\lambda_{1} \nabla u+\lambda_{2} \nabla v+\lambda_{3} \nabla \varphi+\lambda_{4} \nabla a\right]
\end{array}\right) .
$$

Observation model: As for this operator its computation is obvious and we get:

$$
\begin{aligned}
& \left(\partial \mathbb{H}_{\mathbf{v}}\right)^{*}=\nabla I \\
& \left(\partial \mathbb{H}_{\varphi}\right)^{*}=\partial \mathbb{H}_{\varphi}=-\frac{\Lambda-\widehat{\Lambda}}{\sigma_{\Lambda}} \cos (\omega t+\varphi) \\
& \left(\partial \mathbb{H}_{a}\right)^{*}=\partial \mathbb{H}_{a}=-\frac{\Lambda-\widehat{\Lambda}}{\sigma_{\Lambda}}
\end{aligned}
$$

\section{B - NUMERICAL IMPLEMENTATION}

Because of their simplicity of implementation, we decided to use explicit schemes. However, numerical instability in time were observed for forward and backward integrations in algorithm (1) with a standard central finite differencing scheme. We decided to exploit specific explicit Euler schemes [53], [54] adapted to the integration of complex physical models as the ones we are dealing with. It is indeed demonstrated in [53], [54] that such numerical models are conservative, meaning that main physical quantities are kept along the integration process and that discretization errors are bounded. From the adjoint models:

$$
\begin{aligned}
& -\frac{\partial \lambda_{1}}{\partial t}-A_{1}\left[\mathbf{v}^{\mathrm{T}} \nabla \lambda_{1}+v_{y} \lambda_{1}-v_{x} \lambda_{2}-\varphi_{x} \lambda_{3}-a_{x} \lambda_{4}\right]=-I_{x} \mathbb{H}_{\mathbf{v}}(\mathbf{v}, \partial \mathbf{v}), \\
& -\frac{\partial \lambda_{2}}{\partial t}-A_{1}\left[\mathbf{v}^{\mathrm{T}} \nabla \lambda_{2}+u_{x} \lambda_{2}-u_{y} \lambda_{1}-\varphi_{y} \lambda_{3}-a_{y} \lambda_{4}\right]=-I_{y} \mathbb{H}_{\mathbf{v}}(\mathbf{v}, \partial \mathbf{v}), \\
& -\frac{\partial \lambda_{3}}{\partial t}-A_{1}\left[\mathbf{v}^{\mathrm{T}} \nabla \lambda_{3}+u_{x} \lambda_{3}+v_{y} \lambda_{3}\right] \\
& \quad-A_{k} \mathbf{v}^{\mathrm{T}}\left[\lambda_{1} \nabla u+\lambda_{2} \nabla v+\lambda_{3} \nabla \varphi+\lambda_{4} \nabla a\right]=-\left(\partial \mathbb{H}_{a, \varphi}\right)^{*} \mathbb{H}_{a, \varphi}, \\
& -\frac{\partial \lambda_{4}}{\partial t}-A_{1}\left[\mathbf{v}^{\mathrm{T}} \nabla \lambda_{4}+u_{x} \lambda_{4}+v_{y} \lambda_{4}\right] \\
& -k A_{2} \mathbf{v}^{\mathrm{T}}\left[\lambda_{1} \nabla u+\lambda_{2} \nabla v+\lambda_{3} \nabla \varphi+\lambda_{4} \nabla a\right]=-\left(\partial \mathbb{H}_{a, \varphi}\right)^{*} \mathbb{H}_{a, \varphi},
\end{aligned}
$$


and by splitting each equation in two parts, for instance for $\lambda_{1}$ :

$$
\left\{\begin{aligned}
\frac{\partial \lambda_{1}}{\partial t} & =-A_{1}\left[u\left(\lambda_{1}\right)_{x}+v_{y} \lambda_{1}\right], \\
\frac{\partial \lambda_{1}}{\partial t} & =-A_{1}\left[v\left(\lambda_{1}\right)_{y}-\varphi_{x} \lambda_{3}-a_{x} \lambda_{4}\right]+I_{x} \mathbb{H}_{\mathbf{v}}(\mathbf{v}, \partial \mathbf{v}),
\end{aligned}\right.
$$

the explicit Euler numerical scheme gives:

$$
\begin{aligned}
& \left(\lambda_{1}\right)_{i, j}^{k-1}=\left(\lambda_{1}\right)_{i, j}^{k} \\
& \quad-\left(S_{x}\left(\lambda_{1}, u\right)_{i, j}^{k}+\frac{1}{2}\left(v_{i, j+1}^{k}-v_{i, j-1}^{k}\right)\left(\lambda_{1}\right)_{i, j}^{k}\right) \triangle t A_{1} \\
& \left(\lambda_{1}\right)_{i, j}^{k-1}=\left(\lambda_{1}\right)_{i, j}^{k}-\left(S_{y}\left(\lambda_{1}, v\right)_{i, j}^{k}-\frac{1}{2}\left(\varphi_{i+1, j}^{k}-\varphi_{i-1, j}^{k}\right)\left(\lambda_{3}\right)_{i, j}^{k}\right. \\
& \left.-\frac{1}{2}\left(a_{i+1, j}^{k}-a_{i-1, j}^{k}\right)\left(\lambda_{4}\right)_{i, j}^{k}\right) \triangle t A_{1}+\left(I_{x} \mathbb{H}_{\mathbf{v}}(\mathbf{v}, \partial \mathbf{v})\right)_{i, j}^{k}
\end{aligned}
$$

where

$$
S_{x}\left(\lambda_{1}, u\right)_{i, j}^{k}=\max \left(\left(\lambda_{1}\right)_{i, j}^{k}, 0\right)\left(u_{i, j}^{k}-u_{i-1, j}^{k}\right)+\min \left(\left(\lambda_{1}\right)_{i, j}^{k}, 0\right)\left(u_{i+1, j}^{k}-u_{i, j}^{k}\right) .
$$

Note that these numerical schemes are backward since the initial condition for $\lambda_{i}, i=1 \ldots 4$ is given at time $t=T$. Thus, value of $\lambda_{i}$ computed from (24) is used to update $\lambda_{i}$ in (25).

The numerical scheme to compute the incremental variable is obtained by solving the following system of equations:

$$
\frac{\partial \delta \mathcal{X}}{\partial t}+A_{1}\left(\delta \mathbf{v}^{\mathrm{T}} \nabla \mathcal{X}+\mathbf{v}^{\mathrm{T}} \nabla \delta \mathcal{X}\right)+\left(A_{k} \delta \varphi+k A_{2} \delta a\right) \mathbf{v}^{\mathrm{T}} \nabla \mathcal{X}=\mathbf{Q} * \lambda .
$$

The first equation from system of equations (26) can be discretized as follows:

$$
\begin{aligned}
\frac{\partial \delta u}{\partial t}+A_{1}\left(u \delta u_{x}\right. & \left.+v \delta u_{y}+u_{x} \delta u+u_{y} \delta v\right) \\
& +\left(A_{k} \delta \varphi+k A_{2} \delta a\right)\left(u u_{x}+v u_{y}\right) \delta a=\mathbf{Q}_{1} * \lambda_{1}
\end{aligned}
$$

Again, equation (27) is split into two parts:

$$
\left\{\begin{aligned}
\frac{\partial \delta u}{\partial t}+A_{1}\left(u \delta u_{x}\right. & \left.+u_{x} \delta u\right)=0 \\
\frac{\partial \delta u}{\partial t}+A_{1}\left(v \delta u_{y}\right. & \left.+u_{y} \delta v\right) \\
& +\left(A_{k} \delta \varphi+k A_{2} \delta a\right)\left(u u_{x}+v u_{y}\right) \delta a=\mathbf{Q}_{1} * \lambda_{1} .
\end{aligned}\right.
$$

Using the explicit Euler numerical scheme, the incremental variables are updated in the following way:

$$
\begin{aligned}
\delta u_{i, j}^{k+1}= & \delta u_{i, j}^{k}-\triangle t A_{1}\left(S_{x}(\delta u, u)_{i, j}^{k}+\frac{1}{2}\left(u_{i+1, j}^{k}-u_{i-1, j}^{k}\right) \delta u_{i, j}^{k}\right), \\
\delta u_{i, j}^{k+1}= & \delta u_{i, j}^{k}-\triangle t A_{1}\left(S_{y}(\delta u, v)_{i, j}^{k}+\frac{1}{2}\left(u_{i, j+1}^{k}-u_{i, j-1}^{k}\right) \delta v_{i, j}^{k}\right) \\
& -\frac{1}{2} \triangle t u_{i, j}^{k}\left(A_{k} \delta \varphi_{i, j}^{k}+k A_{2} \delta a_{i, j}^{k}\right)\left(u_{i+1, j}^{k}-u_{i-1, j}^{k}\right. \\
& \left.+u_{i, j+1}^{k}-u_{i, j-1}^{k}\right)+\left(\mathbf{Q}_{1} * \lambda_{1}\right)_{i, j}^{k} .
\end{aligned}
$$

The same procedure is applied for the other incremental variables and the complete implementation follows algorithm (1). Note that the numerical schemes are exactly the same for forward and backward models.

\section{ACKNOWLEDGEMENTS}

This work was funded by the 3DSTRAIN project through a grant from the French National Research Agency (ANR), via the "Technologies for health and autonomy" programme, under grant number ANR-11-TecSan002. The authors also thank Patrick Clarysse for his valuable technical assistance on using inTag software.

\section{REFERENCES}

[1] S. Hunt, W. Abraham, C. M.H., A. Feldman, F. G.S., T. Ganiats, M. Jessup, M. Konstam, D. Mancini, K. Michl, J. Oates, P. Rahko, M. Silver, L. Stevenson, and C. Yancy, "2009 focused update incorporated into the ACC/AHA 2005 guidelines for the diagnosis and management of heart failure in adults: a report of the American College of Cardiology Foundation/American Heart Association task force on practice guidelines: developed in collaboration with the International Society for Heart and Lung Transplantation," Circulation, vol. 119, pp. 391-479, 2009. 1

[2] C. Yancy, M. Jessup, B. Bozkurt, J. Butler, D. Casey, M. Drazner, G. Fonarow, S. Geraci, T. Horwich, J. Januzzi, M. Johnson, E. Kasper, L. W.C., F. Masoudi, M. P.E., J. McMurray, J. Mitchell, P. Peterson, B. Riegel, F. Sam, L. Stevenson, W. Tang, E. Tsai, and B. Wilkoff, "2013 ACCF/AHA guideline for the management of heart failure: A report of the American College of Cardiology Foundation/American Heart Association task force on practice guidelines," Journal of the American College of Cardiology, vol. 62(16), pp. 147-239, 2013. 1

[3] S. Raman and O. Simonetti, "The CMR examination in heart failure," Heart Failure Clinics, vol. 5(3), pp. 283-300, 2009. 1

[4] M. Jeung, P. Germain, P. Croisille, S. El ghannudi, C. Roy, and A. Gangi, "Myocardial tagging with MR imaging: overview of normal and pathologic findings," Radiographics, vol. 5(3), pp. 283-300, 2012. 1

[5] N. F. Osman, E. R. McVeigh, and J. L. Prince, "Imaging heart motion using harmonic phase MRI," IEEE Transactions on Medical Imaging, vol. 19(3), pp. 186-202, 2000. 1

[6] T. Arts, F. Prinzen, T. Delhaas, J. Milles, A. Rossi, and P. Clarysse, "Mapping displacement and deformation of the heart with local sinewave modeling," IEEE Transactions on Medical Imaging, vol. 29, pp. 1114-1123, 2010. 1

[7] M. Alessandrini, A. Basarab, H. Liebgott, and O. Bernard, "Myocardial motion estimation from medical images using the monogenic signal," IEEE Transactions on Image Processing, vol. 22(3), pp. 1084-1095, 2013. 1

[8] A. Kono, P. Croisille, T. Nishii, K. Nishiyama, K. Kyotani, M. Shigeru, S. Takamine, S. Fujiwara, and K. Sugimura, "Cardiovascular magnetic resonance tagging imaging correlates with myocardial dysfunction and T2 mapping in idiopathic dilated cardiomyopathy," International Journal of Cardiovascular Imaging, 2014. 1

[9] X. Papademetris, A. J. Sinusas, D. P. Dione, and J. S. Duncan, "Estimation of 3D left ventricular deformation from echocardiography," Medical Image Analysis, vol. 5, pp. 17-28, 2001. 1

[10] A. Elen, H. F. Choi, D. Loeckx, H. Gao, P. Claus, P. Suetens, F. Maes, and J. Dhooge, "Three-dimensional cardiac strain estimation using spatiotemporal elastic registration of ultrasound images: A feasibility study," IEEE Transactions on Medical Imaging, vol. 27, pp. 1580-1591, 2008. 1

[11] M. De-Craene, G. Piella, O. Camara, N. Duchateau, E. Silva, A. Doltra, J. D'hooged, J. Brugada, M. Sitges, and A. F. Frangi, "Temporal diffeomorphic free-form deformation: Application to motion and strain estimation from 3D echocardiography," Medical Image Analysis, vol. 16, pp. 427-450, 2011. 1

[12] T. Mansi, X. Pennec, M. Sermesant, H. Delingette, and N. Ayache, "ilogdemons: A demons-based registration algorithm for tracking incompressible elastic biological tissues," Int J Computer Vision, vol. 92, pp. 92-111, 2011. 1

[13] M. De-Craene, C. Tobon-Gomez, C. Butako, N. Duchateau, G. Piella, K. S. Rhode, and A. F. Frangi, "Temporal diffeomorphic free form deformation (TDFFD) applied to motion and deformation quantification of tagged MRI sequences," in STACOM, Lecture Notes in Computer Science, vol. 7085,2012 , p. 6877.1

[14] V. Tavakoli and N. Sahba, "Cardiac motion and strain detection using 4D CT images: comparison with tagged MRI, and echocardiography," Int J Cardiovasc Imaging, vol. 30, pp. 175-184, 2014. 1

[15] P. Clarysse, C. Basset, L. Khouas, P. Croisille, D. Friboulet, C. Odet, and I. Magnin, "Two-dimensional spatial and temporal displacement and deformation field fitting from cardiac magnetic resonance tagging," Medical Image Analysis, vol. 4, pp. 253-268, 2000. 1

[16] J. Schaerer, C. Casta, J. Pousin, and P. Clarysse, "A dynamic elastic model for segmentation and tracking of the heart in MR image sequences," Medical Image Analysis, vol. 14, pp. 738-749, 2010. 1

[17] C. Tobon-Gomez, M. De-Craene, K. McLeod, T. Lautz, W. Shi, A. Hennemuth, A. Prakosa, H. Wang, G. Carr-White, S. Kapetanakis, A. Lutz, V. Rasche, T. Schaeffter, C. Butakoff, O. Friman, T. Mansi, M. Sermesant, X. Zhuang, S. Ourselin, H. Peitgen, X. Pennec, R. Razavi, 
D. Reuckert, A. Frangi, and K. Rhode, "Benchmarking framework for myocardial tracking and deformation algorithms: An open access database," Medical Image Analysis, vol. 17, pp. 632-648, 2013. 1

[18] H. Wang and A. Amini, "Cardiac motion and deformation recovery from MRI: a review," IEEE Transactions on Medical Imaging, vol. 31, 2012. 1

[19] J. Lions, "Optimal control of systems governed by PDEs," SpringerVerlag, 1971. 1

[20] F.-X. Le Dimet and O. Talagrand, "Variational algorithms for analysis and assimilation of meteorological observations: theoretical aspects," Tellus Series A: Dynamic Meteorology and Oceanography, vol. 38, 1986. $1,3,4$

[21] N. Papadakis and E. Mémin, "A variational technique for time consistent tracking of curves and motion," Journal of Mathematical Imaging and Vision, vol. 31, no. 1, pp. 81-103, 2008. 1, 3

[22] C. Thomas, T. Corpetti, and E. Mémin, "Data assimilation for convective cells tracking on meteorological image sequences," IEEE Transactions on Geoscience and Remote Sensing, vol. 48, pp. 3162-3177, 2010. 1, 3

[23] S. Ba, T. Corpetti, and R. Fablet, "Multi-resolution missing data interpolation in SST image series," in IEEE International Conference on Image Processing, ICIP '11, 1517-1520, Ed., Brussels, Belgium, September 2011. 1

[24] I. Herlin, D. Béréziat, N. Mercier, and S. Zhuk, "Divergence-free motion estimation," in Proc. of Europian Conference on Computer Vision (ECCV), 2012. 1, 2, 3

[25] A. Bennett, Inverse Methods in Physical Oceanography. Cambridge University Press, 1992. 1

[26] O. Talagrand, "Assimilation of observations, an introduction," J. Meteor. Soc. Jap., vol. 75, pp. 191-209, 1997. 1

[27] P. Vidard, E. Blayo, F.-X. Le Dimet, and A. Piacentini, "4D Variational Data Analysis with Imperfect Model," Flow, Turbulence and Combustion, vol. 65, no. 3-4, pp. 489-504, 2000. 1

[28] D. Béréziat and I. Herlin, "Solving ill-posed image processing problems using data assimilation," Numerical Algorithms, 2010. 1

[29] J. Sainte-Marie, D. Chapelle, and M. Sorine, "Data assimilation for an electromechanical model of the myocardium," Second M.I.T. Conference on Computational Fluid and Solid Mechanic, pp. 1801-1804, 2003. 1

[30] M. Sermesant, P. Moireau, O. Camara, J. Sainte-Marie, R. Andriantsimiavona, R. Cimrman, D. L. Hill, D. Chapelle, and R. Razavi, "Cardiac function estimation from MRI using heart model and data assimilation: Advances and difficulties," Medical Image Analysis, vol. 10, pp. 642656, 2006. 1

[31] H. Sundar, Davatzikos, C., and G. Biros, "Biomechanically-constrained 4D estimation of myocardial motion," in Proc. of Medical Image Computing and Computer Assisted Intervention (MICCAI), ser. LNCS, vol. 5762. Springer, 2009, pp. 257-265. 1

[32] H. Delingette, F. Billet, K. C. L.Wong, M. Sermesant, K. Rhode, C. M. Ginks, Aldo Rinaldi, R. Razavi, and A. N., "Personalization of cardiac motion and contractility from images using variational data assimilation," IEEE Transactions on Biomedical Engineering, vol. 10, pp. 20-24, 2012. 1

[33] S. Marchesseau, H. Delingette, M. Sermesant, R. Cabrera-Lozoya, C. Tobon-Gomez, P. Moireau, R. F. i Ventura, K. Lekadir, A. Hernandez, M. Garreau, E. Donal, C. Leclercq, S. Duckett, K. Rhode, C. Rinaldi, A. Frangi, R. Razavi, D. Chapelle, and N. Ayache, "Personalization of a Cardiac Electromechanical Model using Reduced Order Unscented Kalman Filtering from Regional Volumes," Medical Image Analysis, vol. 17, pp. 816-829, 2013. 1

[34] P. Ferreira, P. Gatehouse, R. Mohiaddin, and D. Firmin, "Cardiovascular magnetic resonance artefacts," Journal of Cardiovascular Magnetic Resonance, vol. 15:41, 2013. 2

[35] K. Alfakih, S. Plein, H. Thiele, T. Jones, J. P. Ridgway, and M. U. Sivananthan, "Normal human left and right ventricular dimensions for mri as assessed by turbo gradient echo and steady-state free precession imaging sequences," Journal of Magnetic Resonance Imaging, vol. 17, no. 3, pp. 323-329, 2003. 2

[36] M. D. Cerqueira, N. J. Weissman, V. Dilsiziana, A. K. Jacobs, S. Kaul, W. K. Laskey, D. J. Pennell, J. A. Rumberger, T. Ryan, and M. S. Verani, "Standardized Myocardial Segmentation and Nomenclature for Tomographic Imaging of the Heart: A Statement for Healthcare Professionals from the Cardiac Imaging Committee of the Council on Clinical Cardiology of the American Heart Association," Circulation, vol. 105, pp. 539-542, 2002. 2

[37] T. Corpetti, P. Héas, E. Mémin, and N. Papadakis, "Pressure image assimilation for atmospheric motion estimation," Tellus Series A: Dynamic Meteorology and Oceanography, vol. 61 A(1), pp. 160-178, 2009. 2
[38] B. Lucas and T. Kanade, "An iterative image registration technique with an application to stereo vision," Artificial Intelligence, pp. 674-679, 1981. 4

[39] B. Horn and B. Schunck, "Determining optical flow," Artificial Intelligence, vol. 17, pp. 185-203, 1981. 4

[40] R. Deriche, P. Kornprobst, and G. Aubert, "Optical-flow estimation while preserving its discontinuities: A variational approach," In Proceedings of the second Asian Conference on Computer Vision, vol. 2, pp. 71-80, 1995. 4

[41] T. Brox, A. Bruhn, N. Papenberg, and J. Weickert, "High accuracy optical flow estimation based on a theory for warping," European Conference on Computer Vision (ECCV), vol. 4, pp. 25-36, 2004. 4

[42] T. Corpetti and E. Mémin, "Stochastic uncertainty models for the luminance consistency assumption," IEEE Transactions on Image Processing, vol. 21, pp. 481-493, 2012. 4

[43] P. Perona and J. Malik, "Scale-space and edge detection using anisotropic diffusion," IEEE Transactions on Pattern Analysis and Machine Intelligence, vol. 12, pp. 629-639, 1990. 4

[44] L. Alvarez, P.-L. Lions, and J.-M. Morel, "Image selective smoothing and edge detection by nonlinear diffusion," SIAM Journal on Numerical Analysis, vol. 29, pp. 845-866, 1992. 4

[45] T. David and D. Rachid, "Orthonormal vector sets regularization with PDEs and applications," International Journal of Computer Vision, vol. 50(3), pp. 237-252, 2002. 4

[46] D. Tschumperlé and R. Deriche, "Vector-valued image regularization with PDEs: A common framework for different applications," IEEE Transactions on Pattern Analysis and Machine Intelligence, vol. 27, pp. 506-517, 2005. 4

[47] V. Tuyisenge, A. Albouy-Kissi, L. Cassagnes, E. Coupez, C. Merlin, P. Windyga, and L. Sarry, "Variational myocardial tracking from cineMRI with non-linear regularization," in Proc. of the 10th IEEE Int. Symposium on Biomedical Imaging (ISBI), 2013. 4

[48] V. Tuyisenge, A. Albouy-Kissi, and L. Sarry, "Variational myocardial tracking from cine-MRI with non-linear regularization: Validation of radial displacements vs. tagged-MRI," in Functional Imaging and Modeling of the Heart (FIMH), ser. LNCS, vol. 7945. Springer, 2013, pp. 334-341. 4

[49] J. Weickert, "Anisotropic diffusion in image processing," Stuttgart: Teubner-Verlag, 1998. 4

[50] A. Vidard, V. Chabot, and M. Nodet, "Accounting for correlated observation errors in image data assimilation," in Workshop on correlated observation errors in data assimilation. Reading, United Kingdom: ESA - University of Reading, Apr 2014. [Online]. Available: http://hal.inria.fr/hal-00984508 4

[51] R. F. Duncan, B. K. Dundon, A. J. Nelson, J. Pemberton, K. Williams, M. I. Worthley, A. Zaman, H. Thomas, and S. G. Worthley, "A study of the 16-segment regional wall motion scoring index and biplane simpson rule for the calculation of left ventricular ejection fraction: A comparison with cardiac magnetic resonance imaging," American Society of Echocardiography, vol. 28, pp. 597-604, 2011. 5

[52] N. Schiller, P. Shah, M. Crawford, A. DeMaria, R. Devereux, H. Feigenbaum, H. Gutgesell, N. Reichek, D. Sahn, and I. Schnittger, "Recommendations for quantitation of the left ventricle by two-dimensional echocardiography. American Society of Echocardiography Committee on Standards, Subcommittee on Quantitation of Two-Dimensional Echocardiograms," American Society of Echocardiography, vol. 2, pp. 358-367, 1989. 5

[53] G.-S. Jiang and E. Tadmor, "Non-oscillatory central schemes for multidimensional hyperbolic conservation laws," Journal of Computational Physics, 1997. 10

[54] D. Levy and E. Tadmor, "Non-oscillatory central schemes for the incompressible 2-D euler equations," Mathematical Research Letters, vol. 4, pp. 321-340, 1997. 10 